\title{
Visual hypo and hypergnosia as exemplars of poles of psychic tonus in the occipital lobes: Multiple case analyses
}

\author{
Claude M.J. Braun* and Anik Guimond \\ Institut de Sciences Cognitives and Department of Psychology, Université du Québec à Montréal, Montréal, \\ Canada
}

\begin{abstract}
The "psychic tonus" model or PTM [1] of hemispheric specialization states that the left hemisphere is a psychic and behavioral activator and that the right hemisphere is an inhibitor. The PTM predicts that the tonus of visual representation ought to manifest hemispheric specialization in the occipital lobes. Specifically PTM predicts that pathological positive visual tonus (visual hallucination) ought to be associated more frequently with right occipital lesions. PTM also predicts that pathological negative visual tonus (loss of visual imagery) ought to result more often from left occipital lesions. We reviewed 78 cases of post lesion hallucination and 12 cases of post lesion loss of evocation of images, all following a unilateral lesion. Analyses of these relevant previously published cases support the predictions. In accordance with previously published demonstrations of hemispheric specialization for auditory tonus in the temporal lobes and for somesthetic tonus in the parietal lobes, the present findings extend the psychic tonus model of hemispheric specialization to vision in the occipital lobes.
\end{abstract}

Keywords: Visual representation, hallucination, psychic tonus, hemispheric specialization, hypognosia

\section{Introduction}

\subsection{Psychic tonus: A hemispheric specialization interpretation of positive and negative symptoms}

Braun [1] has developed a model of hemispheric specialization principally based on effects of focal lesions which he has termed "psychic tonus". This model proposes a new framework for interpretation of positive and negative behavioral symptoms. The model draws equally from psychiatry as it does from neurology as well as biopsychology of normal function in humans and animals. The model is based on demonstrations to the effect that psychomotor baseline [2,3], libido [3,4],

\footnotetext{
*Corresponding author: Claude M.J. Braun, $\mathrm{PhD}$, Professeur titulaire, Centre de Neurosciences Cognitives, UQAM, C.P 8888, Succ. Centre-Ville, Montréal, Québec, Canada, H3C 3P8. Tel.: +514 9873000 (4814); Fax: +514 987 8952; E-mail: Braun.Claude@ UQAM.CA; URL: http://www.er.uqam.ca/nobel/r31210/home.html.
}

talkativeness [3,5], immune function [6], memory [7], audition [8] and somesthesia [9] are all modulated in similar opposed ways by the two hemispheres in right handers. The normal left hemisphere increases psychic tonus while the right decreases it.

The model predicts that post lesion visual anomalies should also manifest the same (opposite) dissociation according to lesion side. Left hemisphere lesions should produce negative visual anomalies (dulling of visual representation, i.e., of imagination) because of direct loss of the specialized circuitry of the damaged hemisphere. Right hemisphere lesions should produce positive visual anomalies such as hallucinations due to release of the specialized circuitry of the undamaged hemisphere. It is unusual but not unheard of [10] for dulling or loss of perceptual representations and hallucinations to be considered flip sides of a same coin. To make this point clear we will term positive distortion of perception "hypergnosia" and negative distortion "hypognosia". A mental image can be disturbed in two directions on a continuum. It can be over-excitable too 
lush, too rich, too driven, hypertonic, i.e., hypergnosic on the one hand. However it can be under-excitable, frugal, impoverished, sluggish, hypotonic, i.e., hypognosic on the other. The former, in extreme form, is none other than visual hallucination. The latter, in extreme form, is none other than the loss of visual imagery (in the presence of intact reception sensation and elementary visual perception).

\subsection{Disorders of visual perception}

Short term visual memory and imagination are barely distinguishable constructs. There have been many functional imaging investigations of encoding of visual material, verbal or non verbal. Regarding lateralization of the activations reported, there is a strong constant. The left hemisphere is practically always activated more than the right during encoding into memory (see Cabeza \& Nyberg [11], for a meta-review). Perhaps not surprisingly, this has been many times reported for verbal material. More surprisingly, the same hemispheric asymmetry has consistently been reported for non verbal material as well [11]. Some of this asymmetry has been interpreted as an effect of semantic (verbal) processing which is thought to occur even when participants are encoding non verbal material [11]. Farah and colleagues [12] reviewed a series of case reports of loss of "visual imagery" (in both visual fields) and concluded that the trend was indicative of a higher frequency of left hemisphere lesions. Goldenberg and Artner [13] tested visual imagery and other functions in groups of patients with right and left posterior cerebral artery lesions. They concluded that left hemisphere lesioned patients indeed had poorer visual imagery than right hemisphere lesioned patients but that this was due to a loss of visual knowledge about the objects rather than a loss of imagery per se. The same explanation comes up in reviews of findings to the effect that left hemisphere lesions cause memory impairment, including of non verbal material, far more often than right hemisphere lesions (see Gillespie, Bowen and Foster [14] for a meta-analysis of the literature). However, that argument does not explain why the left hemisphere is found to contribute more to memory than the right in animals (see Braun [1], for a review of that literature). To sum up, functional imaging and the anatomoclinical method both support a strong left hemispheric specialization for visual imagination or encoding into memory. Research with animals demonstrates that this hemispheric asymmetry is not entirely due to verbal-semantic processing during encoding or during imagination.
Visual hallucination is rarer than auditory or somesthetic hallucination, and thus functional imaging studies of visual hallucination are less numerous than of hallucination in those other modalities. Only one study [15] has investigated visual hallucination in large groups of patients with good sets of hallucinatory incidents. Oishi and colleagues [15] compared two groups of patients with Parkinson's disease (PD), a subgroup with visual hallucinations $(\mathrm{N}=24)$ and another without $(\mathrm{N}=41)$ using rCBF. They found right fusiform hypometabolism in the hallucinating group. NaganoSaito and colleagues [16] also compared a few PD patients with and without visual hallucinations. They found left frontal activation in the hallucinating cases. O'Brian and colleagues [17] studied hallucination in a few patients with Lewy body dementia (LBD) using SPECT. They reported a correlation between left cingulate and parietal hypometabolism and visual hallucinations. Imamura and colleagues [18] carried out a similar study and reported right parietal and temporal activation in the hallucinating cases, not significant when controlled for multiple comparisons. Mori and colleagues [19] reported a similar study as well, and found no evidence of lateralization of activations. In these latter three studies of LBD there seemed to be very few hallucinating cases, and very few hallucinatory incidents, suggesting to us that these results should not be judged informative for hemispheric specializations in the normal brain. Adachi and colleagues [20] studied visual hallucination in five patients with Charles Bonnet syndrome (cases without focal brain lesions). Importantly, SPECT measurements were obtained when patients were and were not hallucinating. Hallucinations were clearly related to activations, particularly in the temporal lobes, but these activations occurred equally often in either hemisphere. Shedlack and colleagues [21] found MRI stigmata in 12 elderly Charles Bonnet patients, but there was no evidence of any asymmetry of the stigmata. Braun and colleagues [22] reviewed single case reports of post lesion visual hallucination and found that of 55 cases due to a unilateral brain lesion 35 presented a right hemisphere lesion $(p<0.05)$. In that review the intrahemispheric location of the lesion was unconstrained and the field in which hallucinations were experienced was not noted. To sum up, the functional imaging and lesion literatures on visual hallucination are not yet very informative about occipital lateralization, but the most credible evidence available $[15,22]$ suggests a source weakly lateralized to the left hemisphere. 


\subsection{Statement of purpose}

This project proposes to optimize exploitation of multiple case analysis to test the idea according to which hemispherically specialized psychic tonus would be a good explanation of hypergnosia and hypognosia in the visual modality expressed exclusively in the occipital lobes. To this end carefully selected unilateral post-lesion cases were reviewed using a classification system appropriate for the inference test foreseen at outset.

\section{Method}

Cases sought here were 1) non paroxystic cases of visual hallucination (in one or both fields) due to a unilateral lesion involving the occipital lobe 2) non paroxystic cases of selective loss of visual imagery in both fields in the awake subject due to a unilateral lesion involving the occipital lobe. All cases manifesting any epileptic sign (seizures or twitching or drop attacks or EEG paroxysm or evidence of successful control of the hallucinations with anticonvulsants) were excluded from this study because hallucinations can come from an ipsilesional paroxysm even in presence of a massive lesion (see [23] for an excellent demonstration of the latter). Manifestation of the target symptom of both groups was an exclusion criterion. EEG findings, presence of aphasia, the field in which the hallucination occurred, the simple (unformed) versus complex (formed) nature of the hallucination, evidence of obvious episodic memory content in the hallucintation, patient age, gender and hand preference, lesion location and etiology of the lesion, were all noted systematically for each case, in view of control analyses.

Each of these data bases will be submitted to a same conservative non parametric inference test (two tailed $\mathrm{Chi}^{2}$ test) of preponderance of right or left sided lesions. Right hemisphere lesions are expected to produce hypergnosia (hallucinations) and left hemisphere lesions are expected to produce hypognosia (loss of imagination). As a side issue, we were interested in indirectly testing the idea according to which perilesional (i.e., ipsilesional) hypermetabolism could explain unilateral hallucinations. Perilesional hypermetabolism has been reported in several cases of unilateral hallucination [24-26], including selectively during hallucination [27-29]. In such cases, hallucinations are occasionally suspected of being irritative and paroxystic in nature [27]. However, ipsilesional hypometabolism has been more frequently reported [30-35], including selectively during the hallucinations $[36,37]$. In such cases, hallucinations are not (or are less) suspected of resulting from paroxystic activity. Contralesional hypermetabolism has been reported in a case with visual hallucinations [38], but this seems to be an unusual finding. We reasoned that if the perilesional release account of post lesion hallucination in a single field is the best explanation, then the majority of cases of unilateral hallucination should manifest their hallucinations in the contralesional (usually partially anopic) field, as noted initially by Kolmel in his review of 16 patients [39]. Given as much, we were interested in determining whether a psychic tonus effect (i,e., a lesion side effect), in the cases with hallucination, would be limited to cases with bilateral hallucinations, with complex hallucinations or with hallucinations laden with content from episodic memory, all more apt to reflect higher order hemispheric specialization. Though we suppose that a hemispheric specialization effect ought to be due to contralesional release, we planned to test that notion, if possible, with EEG findings (i.e., ipsilesional slowing) and cases with SPECT imaging.

\section{Results}

\subsection{Positive disorders of visual perception}

Cases with post-lesion visual hallucination were sought by means of multitudes of medline and google scholar searches and cross referencing from related readings. For purposes of testing the psychic tonus model, cases with hallucinations in both visual fields are in principle more interesting than cases with hallucinations in a single field. Indeed to the extent that such a specific experience as hallucination can be conceived as a peculiar manifestation of unbridled mentation, i.e., elevated mental tonus, then the hallucination is all the more plausibly interpreted as such if it invades both visual fields - precluding its interpretation as a banal contralesional effect. Along the same line of reasoning, cases with complex hallucinations and/or hallucinated elements drawn from episodic memory (a hallucination of one's mother for example) are more relevant in principle for testing the psychic tonus model because these phenomena more readily reflect a "general" mental state than a small unformed hallucination (e.g., a spot of light) occurring in the anoptic field. Also, cases with comfirmed slowing of EEG over the lesion and by the same stroke rejection of the hypothesis of an irrita- 
Table 1

Non-paroxystic visual hallucinations in both visual fields following a radiologically or surgically objectified unilateral occipital lobe lesion

\begin{tabular}{|c|c|c|c|c|c|}
\hline $\begin{array}{l}\text { Age at onset } \\
\text { gender and } \\
\text { hand preference }\end{array}$ & $\begin{array}{l}\text { Locus of } \\
\text { the lesion }\end{array}$ & $\begin{array}{l}\text { Lesion } \\
\text { etiology }\end{array}$ & $\begin{array}{l}\text { Type of hallucination and location } \\
\text { in the visual field(s) } \\
*=\text { the hallucinated image is from } \\
\text { episodic memory }\end{array}$ & Clinical considerations & Reference \\
\hline $\begin{array}{l}\text { 71 Female } \\
\mathrm{H} \text { ? }\end{array}$ & $\begin{array}{l}\text { Right } \\
\text { occipital }\end{array}$ & Infarct & $\begin{array}{l}\text { Complex visual hallucinations* } \\
\text { in both visual fields }\end{array}$ & $\begin{array}{l}\text { No EEG, seizures or psychiatric } \\
\text { comorbidity reported }\end{array}$ & $\begin{array}{l}{[61]} \\
\text { Michel } \\
\& \text { Troost }\end{array}$ \\
\hline $\begin{array}{l}73 \\
\text { Female } \\
\text { H? }\end{array}$ & $\begin{array}{l}\text { Right } \\
\text { occipito- } \\
\text { temporal }\end{array}$ & Infarct & $\begin{array}{l}\text { Complex visual hallucinations* } \\
\text { in both visual fields }\end{array}$ & $\begin{array}{l}\text { No EEG, seizures or psychiatric } \\
\text { comorbidity reported }\end{array}$ & $\begin{array}{l}{[62]} \\
\text { Meadows } \\
\& \text { Munro }\end{array}$ \\
\hline $\begin{array}{l}66 \text { Female } \\
\mathrm{H} \text { ? }\end{array}$ & $\begin{array}{l}\text { Right } \\
\text { occipital }\end{array}$ & Infarct & $\begin{array}{l}\text { Complex visual hallucinations } \\
\text { in both visual fields }\end{array}$ & $\begin{array}{l}\text { No EEG or seizures reported, } \\
\text { reactive depression, psychosis, } \\
\text { paranoia and delusions }\end{array}$ & $\begin{array}{l}{[63]} \\
\text { Mancusa } \\
\& \text { Cole }\end{array}$ \\
\hline $\begin{array}{l}70 \text { Female } \\
\mathrm{H} \text { ? }\end{array}$ & $\begin{array}{l}\text { Left } \\
\text { occipital }\end{array}$ & Infarct & $\begin{array}{l}\text { Complex visual hallucinations } \\
\text { in both visual fields }\end{array}$ & $\begin{array}{l}\text { No EEG, seizures or psychiatric } \\
\text { comorbidity reported }\end{array}$ & $\begin{array}{l}{[64]} \\
\text { Lance }\end{array}$ \\
\hline $\begin{array}{l}75 \text { Male } \\
\mathrm{H} ?\end{array}$ & $\begin{array}{l}\text { Left } \\
\text { occipital }\end{array}$ & Infarct & $\begin{array}{l}\text { Complex visual hallucinations* } \\
\text { in both visual fields }\end{array}$ & $\begin{array}{l}\text { No EEG, seizures or psychiatric } \\
\text { comorbidity reported }\end{array}$ & $\begin{array}{l}{[39]} \\
\text { Kolmel }\end{array}$ \\
\hline $\begin{array}{l}72 \text { Female } \\
\text { RH }\end{array}$ & $\begin{array}{l}\text { Right } \\
\text { occipital }\end{array}$ & Infarct & $\begin{array}{l}\text { Complex visual hallucinations, } \\
\text { apparently in both visual fields }\end{array}$ & $\begin{array}{l}\text { Four EEGs revealed no paroxysmal } \\
\text { activity, only slowing over the lesion, } \\
\text { no seizures were observed, pre-stroke } \\
\text { psychiatric status was normal }\end{array}$ & $\begin{array}{l}{[65]} \\
\text { Peroutka } \\
\text { et al. }\end{array}$ \\
\hline $\begin{array}{l}77 \text { Male } \\
\text { RH }\end{array}$ & $\begin{array}{l}\text { Right } \\
\text { occipital }\end{array}$ & Infarct & $\begin{array}{l}\text { Complex visual hallucinations } \\
\text { in both visual fields }\end{array}$ & $\begin{array}{l}\text { No EEG, seizures or psychiatric } \\
\text { comorbidity reported }\end{array}$ & $\begin{array}{l}{[66]} \\
\text { Dodd } \\
\text { et al. }\end{array}$ \\
\hline $\begin{array}{l}58 \text { Male } \\
\mathrm{H} \text { ? }\end{array}$ & $\begin{array}{l}\text { Right } \\
\text { occipito- } \\
\text { temporo- } \\
\text { parietal }\end{array}$ & $\begin{array}{l}\text { Cerebro- } \\
\text { vascular } \\
\text { accident }\end{array}$ & $\begin{array}{l}\text { Complex visual hallucinations } \\
\text { in both visual fields }\end{array}$ & $\begin{array}{l}\text { Slowing of EEG on left, no mention } \\
\text { of seizures or of previous } \\
\text { psychiatric morbidity }\end{array}$ & $\begin{array}{l}{[67]} \\
\text { Medina } \\
\text { et al. }\end{array}$ \\
\hline $\begin{array}{l}37 \text { Male } \\
\text { RH }\end{array}$ & $\begin{array}{l}\text { Left } \\
\text { occipito- } \\
\text { temporal }\end{array}$ & Infarct & $\begin{array}{l}\text { Complex visual hallucinations* } \\
\text { in both visual fields agitation } \\
\text { and aggressiveness }\end{array}$ & $\begin{array}{l}\text { Slowing of EEG on left, agitation } \\
\text { and aggressiveness }\end{array}$ & $\begin{array}{l}{[67]} \\
\text { Medina } \\
\text { et al. }\end{array}$ \\
\hline $\begin{array}{l}42 \text { Male } \\
\mathrm{H} \text { ? }\end{array}$ & $\begin{array}{l}\text { Left } \\
\text { occipital }\end{array}$ & Abcess & $\begin{array}{l}\text { Complex visual hallucinations* } \\
\text { in both visual fields of people he } \\
\text { had seen before }\end{array}$ & $\begin{array}{l}\text { No EEG, seizures or psychiatric } \\
\text { comorbidity mentioned }\end{array}$ & $\begin{array}{l}{[68]} \\
\text { Lunardi } \\
\text { et al. }\end{array}$ \\
\hline $\begin{array}{l}66 \text { Male } \\
\mathrm{H} \text { ? }\end{array}$ & $\begin{array}{l}\text { Right } \\
\text { occipital }\end{array}$ & Abcess & $\begin{array}{l}\text { Complex visual hallucination* } \\
\text { of objects (apparently in both } \\
\text { fields) }\end{array}$ & $\begin{array}{l}\text { No EEG, seizures or psychiatric } \\
\text { comorbidity mentioned }\end{array}$ & $\begin{array}{l}{[69]} \\
\text { Patterson } \\
\text { et al. }\end{array}$ \\
\hline $\begin{array}{l}53 \text { Male } \\
\text { RH }\end{array}$ & $\begin{array}{l}\text { Right } \\
\text { occipito- } \\
\text { parietal }\end{array}$ & Glioblastoma & $\begin{array}{l}\text { Complex visual perseveration } \\
\text { (palinopsia)* in both fields of } \\
\text { objects seen over the previous } \\
\text { half hour }\end{array}$ & $\begin{array}{l}\text { No EEG, seizures or psychiatric } \\
\text { comorbidity mentioned }\end{array}$ & $\begin{array}{l}{[70]} \\
\text { Maillot } \\
\text { et al. }\end{array}$ \\
\hline $\begin{array}{l}79 \text { Female } \\
\mathrm{H} \text { ? }\end{array}$ & $\begin{array}{l}\text { Right } \\
\text { occipital }\end{array}$ & $\begin{array}{l}\text { Intracranial } \\
\text { heamatoma }\end{array}$ & $\begin{array}{l}\text { Complex visual hallucinations in } \\
\text { both visual fields }\end{array}$ & $\begin{array}{l}\text { No EEG, seizures or psychiatric } \\
\text { comorbidity mentioned }\end{array}$ & $\begin{array}{l}{[71]} \\
\text { Mukaetova- } \\
\text { Ladinsk } \\
\text { et al. }\end{array}$ \\
\hline $\begin{array}{l}47 \text { Female } \\
\mathrm{H} \text { ? }\end{array}$ & $\begin{array}{l}\text { Right } \\
\text { occipital }\end{array}$ & Infarct & $\begin{array}{l}\text { Complex visual hallucinations in } \\
\text { both visual fields }\end{array}$ & $\begin{array}{l}\text { No EEG, seizures or psychiatric } \\
\text { comorbidity mentioned }\end{array}$ & $\begin{array}{l}{[72]} \\
\text { Strandgaard } \\
\text { et al. }\end{array}$ \\
\hline $\begin{array}{l}68 \text { Male } \\
\mathrm{H} \text { ? }\end{array}$ & $\begin{array}{l}\text { Left } \\
\text { occipital }\end{array}$ & Infarct & $\begin{array}{l}\text { Complex visual hallucinations* } \\
\text { in both visual fields (central vision) }\end{array}$ & $\begin{array}{l}\text { No EEG, seizures or psychiatric } \\
\text { comorbidity reported }\end{array}$ & $\begin{array}{l}{[61]} \\
\text { Michel } \\
\text { \& Troost }\end{array}$ \\
\hline $\begin{array}{l}66 \text { Male } \\
\mathrm{H} \text { ? }\end{array}$ & $\begin{array}{l}\text { Right } \\
\text { occipital }\end{array}$ & Glioblastoma & $\begin{array}{l}\text { Complex visual perseveration } \\
\text { (palinopsia)* in both visual fields } \\
\text { of objects and people }\end{array}$ & $\begin{array}{l}\text { Normal EEG, no psychiatric } \\
\text { comorbidity reported }\end{array}$ & $\begin{array}{l}{[73]} \\
\text { Lazaro }\end{array}$ \\
\hline
\end{tabular}


Table 1, continued

\begin{tabular}{|c|c|c|c|c|c|}
\hline $\begin{array}{l}\text { Age at onset } \\
\text { gender and } \\
\text { hand preference }\end{array}$ & $\begin{array}{l}\text { Locus of } \\
\text { the lesion }\end{array}$ & $\begin{array}{l}\text { Lesion } \\
\text { etiology }\end{array}$ & $\begin{array}{l}\text { Type of hallucination and location } \\
\text { in the visual field(s) } \\
*=\text { the hallucinated image is from } \\
\text { episodic memory }\end{array}$ & Clinical considerations & Reference \\
\hline $\begin{array}{l}64 \text { Male } \\
\text { RH }\end{array}$ & $\begin{array}{l}\text { Right } \\
\text { occipito- } \\
\text { temporal }\end{array}$ & Ischemia & $\begin{array}{l}\text { Simple visual hallucinations } \\
\text { (metamorphopsia) in both visual } \\
\text { fields mild prosopagnosia }\end{array}$ & $\begin{array}{l}\text { Slowing of EEG at posterior right } \\
\text { scalp, no psychiatric comorbidity } \\
\text { reported }\end{array}$ & $\begin{array}{l}{[32]} \\
\text { Hoksbergen } \\
\text { et al. }\end{array}$ \\
\hline $\begin{array}{l}62 \text { Male } \\
\mathrm{H} \text { ? }\end{array}$ & $\begin{array}{l}\text { Right } \\
\text { occipito- } \\
\text { parietal }\end{array}$ & Infarct & $\begin{array}{l}\text { Simple vusual hallucinations } \\
\text { "dazzle" in central fields }\end{array}$ & $\begin{array}{l}\text { No EEG, seizures or psychiatric } \\
\text { comorbidity mentioned }\end{array}$ & $\begin{array}{l}{[74]} \\
\text { Landis } \\
\text { et al. }\end{array}$ \\
\hline $\begin{array}{l}20 \text { Male } \\
\mathrm{H} \text { ? }\end{array}$ & $\begin{array}{l}\text { Left } \\
\text { occipital }\end{array}$ & $\begin{array}{l}\text { Penetrating } \\
\text { wound }\end{array}$ & $\begin{array}{l}\text { Simple visual hallucinations } \\
\text { (polyopsia) in both visual fields }\end{array}$ & $\begin{array}{l}\text { No EEG or seizures reported, } \\
\text { personality and psychological } \\
\text { tests failed to disclose significant } \\
\text { neurotic trends }\end{array}$ & $\begin{array}{l}{[75]} \\
\text { Bender }\end{array}$ \\
\hline $\begin{array}{l}56 \text { Male } \\
\mathrm{H} \text { ? }\end{array}$ & $\begin{array}{l}\text { Right } \\
\text { occipital }\end{array}$ & $\begin{array}{l}\text { Metastatic } \\
\text { tumor }\end{array}$ & $\begin{array}{l}\text { Simple visual perseveration } \\
\text { (palinopsia)* in both visual fields }\end{array}$ & $\begin{array}{l}\text { Normal EEG, no mention of } \\
\text { seizures or of psychiatric } \\
\text { comorbidity }\end{array}$ & $\begin{array}{l}{[76]} \\
\text { Estefania } \\
\text { et al. }\end{array}$ \\
\hline
\end{tabular}

tive paroxystic perilesional source of the hallucination are in principle more interesting for a test of the psychic tonus model than cases without EEG.

The ideal post lesion case for testing the psychic tonus model would have a clearly unilateral lesion limited to the occipital lobe (the visual cortex), only one symptom (visual hallucination), the hallucination would be complex, would contain elements from episodic memory, and would deploy in both visual fields, the patient would be right handed, he or she would present no signs of seizures only slowing over the lesion in EEG and no paroxysmal activity, and he or she would not be too young nor too old. It is needless to say that such perfect cases (for a test of the theoretical model under consideration) of course are rather rare. At any rate all these considerations are explicitly accounted for and regrouped accordingly in the next tables -as far as the information was available in each case report.

Of the 78 cases of visual hallucination following a unilateral occipital lesion $48(62 \%)$ had a right hemisphere lesion and $30(38 \%)$ had a left hemisphere lesion. A Chi ${ }^{2}$ test of probability indicates that this difference is significant $\left(\mathrm{Chi}^{2}=4.2, p<0.043\right.$ two tailed) if one were to suppose that each hemisphere is equally at risk for a lesion (see Tables 1, 2 and 3). These results support the psychic tonus model.

We believe left and right hemisphere lesions indeed have equiprobable chances of occuring and of being selected for single case reports (in the absence of selection for type of visual disturbance). Duval and her colleagues [40] assembled 725 cases using similar inclusion/exclusion criteria as here, except that they were not selected for any particular cognitive or psy- chiatric problem (an IQ test was required). This is the largest such data base we are aware of. In that data base there were 387 patients with left hemisphere lesions and 338 with right hemisphere lesions (binomial probability against equiprobability: $p=\mathrm{NS}$, two tailed). Thus it can basically be assumed that when unselected for memory disorder type, the two lesion sides may be considered theoretically equiprobable (or very close to that) when drawn from previously published reports.

\subsection{Negative distortions of visual perception}

Focal brain lesions can affect visual tonus negatively. The best example in the current story line would be a weakening of one's ability to evoke a visual representation from immediate memory. This loss of internal visual evocation is none other than loss of the imaginative function, i.e., loss of imagination or "mind's eye". For purposes of replicability and clarity of our venture we consider a pure loss of visual evocation in the awake mind as an inability to retain in short term memory a visual stimulus which could be properly described and/or drawn in presence of the stimulus. This represents something like the opposite of visual hallucination. There are very few cases of this function being selectively destroyed by a focal unilateral lesion. Some cases present signs of associative agnosia (problems of verbal description of the object) or other aphasic signs or imperfect drawing of the object at copy.

We collected all previously published cases that we could find of selective loss of visual imagery due to a unilateral lesion, specifically, loss of the ability to non verbally evoke an image in immediate memory while 
Table 2

Non-paroxystic visual hallucinations limited to a single visual field following a radiologically or surgically objectified unilateral occipital lobe lesion

\begin{tabular}{|c|c|c|c|c|c|}
\hline $\begin{array}{l}\text { Age at onset } \\
\text { gender and } \\
\text { hand preference }\end{array}$ & $\begin{array}{l}\text { Locus of } \\
\text { the lesion }\end{array}$ & Lesion etiology & $\begin{array}{l}\text { Type of hallucination and location } \\
\text { in the visual field(s) } \\
*=\text { the hallucinated image is from } \\
\text { episodic memory }\end{array}$ & Clinical considerations & Reference \\
\hline $\begin{array}{l}45 \text { Male } \\
\mathrm{H} \text { ? }\end{array}$ & $\begin{array}{l}\text { Left } \\
\text { occipio- } \\
\text { parietal }\end{array}$ & Meningioma & $\begin{array}{l}\text { Complex long lasting hallucina- } \\
\text { tions, including palinoptic* in the } \\
\text { contralesional visual field }\end{array}$ & $\begin{array}{l}\text { No EEG, seizures or psychiatric } \\
\text { comorbidity mentioned }\end{array}$ & $\begin{array}{l}{[77]} \\
\text { Mooney } \\
\text { et al. }\end{array}$ \\
\hline $\begin{array}{l}66 \text { Male } \\
\text { M? }\end{array}$ & $\begin{array}{l}\text { Right } \\
\text { occipital }\end{array}$ & Infarct & $\begin{array}{l}\text { Complex visual hallucinations* } \\
\text { in the contralesional visual field }\end{array}$ & $\begin{array}{l}\text { No EEG, seizures or psychiatric } \\
\text { comorbidity mentioned }\end{array}$ & $\begin{array}{l}{[61]} \\
\text { Michel } \\
\text { \& Troost }\end{array}$ \\
\hline $\begin{array}{l}66 \text { Female } \\
\text { RH }\end{array}$ & $\begin{array}{l}\text { Right } \\
\text { occipital }\end{array}$ & $\begin{array}{l}\text { Infarct }+ \\
\text { atrophy right } \\
\text { temporal }\end{array}$ & $\begin{array}{l}\text { Complex visual hallucinations } \\
\text { in the contralesional visual field }\end{array}$ & $\begin{array}{l}\text { EEG slowing over the lesion, } \\
\text { no psychiatric comorbidity } \\
\text { reported }\end{array}$ & $\begin{array}{l}{[39]} \\
\text { Kolmel }\end{array}$ \\
\hline $\begin{array}{l}65 \text { Female } \\
\mathrm{H} \text { ? }\end{array}$ & $\begin{array}{l}\text { Right } \\
\text { occipital }\end{array}$ & Infarct & $\begin{array}{l}\text { Complex visual hallucinations* } \\
\text { in the contralesional visual field }\end{array}$ & $\begin{array}{l}\text { No EEG, seizures or psychiatric } \\
\text { comorbidity mentioned }\end{array}$ & $\begin{array}{l}{[64]} \\
\text { Lance }\end{array}$ \\
\hline $\begin{array}{l}53 \text { Male } \\
\mathrm{H} \text { ? }\end{array}$ & $\begin{array}{l}\text { Right } \\
\text { occipital }\end{array}$ & Infarct & $\begin{array}{l}\text { Complex visual hallucinations* } \\
\text { in the contralesional visual field }\end{array}$ & $\begin{array}{l}\text { No EEG, seizures or psychiatric } \\
\text { comorbidity mentioned }\end{array}$ & $\begin{array}{l}{[64]} \\
\text { Lance }\end{array}$ \\
\hline $\begin{array}{l}73 \text { Female } \\
\mathrm{H} \text { ? }\end{array}$ & $\begin{array}{l}\text { Right } \\
\text { occipital }\end{array}$ & Infarct & $\begin{array}{l}\text { Complex visual hallucinations* } \\
\text { in the contralesional visual field }\end{array}$ & $\begin{array}{l}\text { No EEG, seizures or psychiatric } \\
\text { comorbidity mentioned }\end{array}$ & $\begin{array}{l}{[64]} \\
\text { Lance }\end{array}$ \\
\hline $\begin{array}{l}72 \text { Male } \\
\mathrm{H} \text { ? }\end{array}$ & $\begin{array}{l}\text { Right } \\
\text { occipito- } \\
\text { parietal }\end{array}$ & Infarct & $\begin{array}{l}\text { Complex visual hallucinations } \\
\text { in the contralesional visual field }\end{array}$ & $\begin{array}{l}\text { Normal EEG, no seizures or } \\
\text { psychiatric comorbidity reported }\end{array}$ & $\begin{array}{l}{[64]} \\
\text { Lance }\end{array}$ \\
\hline $\begin{array}{l}74 \text { Female } \\
\mathrm{H} \text { ? }\end{array}$ & $\begin{array}{l}\text { Right } \\
\text { occipito- } \\
\text { parietal }\end{array}$ & Infarct & $\begin{array}{l}\text { Complex visual hallucinations, } \\
\text { including heutoscopic*, in the } \\
\text { contralesional visual field }\end{array}$ & $\begin{array}{l}\text { No EEG, seizures or psychiatric } \\
\text { comorbidity mentioned }\end{array}$ & $\begin{array}{l}{[39]} \\
\text { Kolmel }\end{array}$ \\
\hline $\begin{array}{l}48 \text { Male } \\
\mathrm{H} \text { ? }\end{array}$ & $\begin{array}{l}\text { Left } \\
\text { occipital }\end{array}$ & Infarct & $\begin{array}{l}\text { Complex visual hallucinations } \\
\text { in the contralesional visual field }\end{array}$ & $\begin{array}{l}\text { No EEG, seizures or psychiatric } \\
\text { comorbidity mentioned }\end{array}$ & $\begin{array}{l}{[64]} \\
\text { Lance }\end{array}$ \\
\hline $\begin{array}{l}64 \text { Male } \\
\mathrm{H} \text { ? }\end{array}$ & $\begin{array}{l}\text { Left } \\
\text { occipital }\end{array}$ & Infarct & $\begin{array}{l}\text { Complex visual hallucinations } \\
\text { in the contralesional visual field }\end{array}$ & $\begin{array}{l}\text { No EEG, seizures or psychiatric } \\
\text { comorbidity mentioned }\end{array}$ & $\begin{array}{l}{[78]} \\
\text { Benson } \\
\text { \& Rennie }\end{array}$ \\
\hline $\begin{array}{l}56 \text { Male } \\
\mathrm{H} \text { ? }\end{array}$ & $\begin{array}{l}\text { Left } \\
\text { occipital }\end{array}$ & Infarct & $\begin{array}{l}\text { Complex persistent visual halluc- } \\
\text { inations in the contralesional } \\
\text { visual field }\end{array}$ & $\begin{array}{l}\text { No EEG, seizures or psychiatric } \\
\text { comorbidity mentioned }\end{array}$ & $\begin{array}{l}{[79]} \\
\text { Kasten } \\
\text { et al. }\end{array}$ \\
\hline $\begin{array}{l}70 \text { Female } \\
\mathrm{H} \text { ? }\end{array}$ & $\begin{array}{l}\text { Left } \\
\text { occipital }\end{array}$ & Infarct & $\begin{array}{l}\text { Complex visual hallucinations } \\
\text { in the contralesional visual field }\end{array}$ & $\begin{array}{l}\text { No EEG, seizures or psychiatric } \\
\text { comorbidity mentioned }\end{array}$ & $\begin{array}{l}{[64]} \\
\text { Lance }\end{array}$ \\
\hline $\begin{array}{l}68 \text { Male } \\
\text { RH }\end{array}$ & $\begin{array}{l}\text { Left } \\
\text { occipito- } \\
\text { parietal }\end{array}$ & $\begin{array}{l}\text { Oligodendro- } \\
\text { glioma }\end{array}$ & $\begin{array}{l}\text { Complex visual hallucinations } \\
\text { in the contralesional visual field }\end{array}$ & $\begin{array}{l}\text { No EEG, seizures or psychiatric } \\
\text { comorbidity mentioned }\end{array}$ & $\begin{array}{l}{[39]} \\
\text { Kolmel }\end{array}$ \\
\hline $\begin{array}{l}50 \text { Female } \\
\text { RH }\end{array}$ & $\begin{array}{l}\text { Left } \\
\text { occipito- } \\
\text { parietal }\end{array}$ & $\begin{array}{l}\text { Resected } \\
\text { glioblastoma } \\
\text { multiform }\end{array}$ & $\begin{array}{l}\text { Complex visual hallucinations } \\
\text { in the contralesional field }\end{array}$ & $\begin{array}{l}\text { No EEG or convulsion noted } \\
\text { except once prior to resection, no } \\
\text { psychiatric comorbidity reported }\end{array}$ & $\begin{array}{l}{[80]} \\
\text { Freiman } \\
\text { et al. }\end{array}$ \\
\hline $\begin{array}{l}59 \text { Female } \\
\text { RH }\end{array}$ & $\begin{array}{l}\text { Right } \\
\text { occipital }\end{array}$ & Stroke & $\begin{array}{l}\text { Complex visual hallucinations, } \\
\text { including palinoptic*, in the } \\
\text { contralesional field }\end{array}$ & $\begin{array}{l}\text { Normal EEG (few independent } \\
\text { sharp waves) no convulsions } \\
\text { mentioned, no psychiatric } \\
\text { comorbidity reported, right } \\
\text { hypometabolism on SPECT }\end{array}$ & $\begin{array}{l}{[35]} \\
\text { Sun } \\
\& \text { Lin }\end{array}$ \\
\hline $\begin{array}{l}64 \text { Female } \\
\text { RH }\end{array}$ & $\begin{array}{l}\text { Right } \\
\text { occipital }\end{array}$ & $\begin{array}{l}\text { Resected tumor } \\
\text { adenocarcinoma }\end{array}$ & $\begin{array}{l}\text { Complex visual hallucinations* } \\
\text { in the contralesional field }\end{array}$ & $\begin{array}{l}\text { Absence of seizures is insisted } \\
\text { upon by the authors but no EEG } \\
\text { was carried out, there were no } \\
\text { hallucinations prior to resection, } \\
\text { past history of anxiety and depr- } \\
\text { ession }\end{array}$ & $\begin{array}{l}{[81]} \\
\text { Faber } \\
\text { \& Johnson }\end{array}$ \\
\hline $\begin{array}{l}49 \text { Male } \\
\mathrm{H} ?\end{array}$ & $\begin{array}{l}\text { Right } \\
\text { occipito- } \\
\text { parietal }\end{array}$ & Excised tumor & $\begin{array}{l}\text { Complex hallucinations (persons, } \\
\text { objects) in the contralesional } \\
\text { visual field }\end{array}$ & $\begin{array}{l}\text { EEG slowing over the lesion, } \\
\text { no evidence of paroxysms, right } \\
\text { hypermetabolism in SPECT }\end{array}$ & $\begin{array}{l}{[26]} \\
\text { Vogeley }\end{array}$ \\
\hline
\end{tabular}


Table 2, continued

\begin{tabular}{|c|c|c|c|c|c|}
\hline $\begin{array}{l}\text { Age at onset } \\
\text { gender and } \\
\text { hand preference }\end{array}$ & $\begin{array}{l}\text { Locus of } \\
\text { the lesion }\end{array}$ & Lesion etiology & $\begin{array}{l}\text { Type of hallucination and location } \\
\text { in the visual field(s) } \\
*=\text { the hallucinated image is from } \\
\text { episodic memory }\end{array}$ & Clinical considerations & Reference \\
\hline $\begin{array}{l}65 \text { Male } \\
\mathrm{H} \text { ? }\end{array}$ & $\begin{array}{l}\text { Right } \\
\text { occipital }\end{array}$ & $\begin{array}{l}\text { Arterio } \\
\text { venous } \\
\text { malformation }\end{array}$ & $\begin{array}{l}\text { Complex visual hallucinations } \\
\text { in the contralesional field }\end{array}$ & $\begin{array}{l}\text { EEG indicates no seizures, } \\
\text { only supralesional slowing, } \\
\text { no psychiatric comorbidity } \\
\text { reported, SPECT indicated } \\
\text { right hypometabolism } \\
\text { during the hallucinations }\end{array}$ & $\begin{array}{l}\text { [37] } \\
\text { Inafuku } \\
\text { et al. }\end{array}$ \\
\hline $\begin{array}{l}73 \text { Female } \\
\mathrm{H} \text { ? }\end{array}$ & $\begin{array}{l}\text { Left } \\
\text { occipito- } \\
\text { temporal }\end{array}$ & Infarct & $\begin{array}{l}\text { Complex visual hallucinations* } \\
\text { in the contralesional field }\end{array}$ & $\begin{array}{l}\text { No epileptic signs in EEG, } \\
\text { phenytoin before and during } \\
\text { hallucinations did not prevent } \\
\text { them, left hypoperfusion in } \\
\text { SPECT }\end{array}$ & $\begin{array}{l}{[82]} \\
\text { Waragai } \\
\text { et al. }\end{array}$ \\
\hline $\begin{array}{l}76 \text { Male } \\
\text { RH }\end{array}$ & $\begin{array}{l}\text { Right } \\
\text { occipital }\end{array}$ & Infarct & $\begin{array}{l}\text { Complex visual hallucinations } \\
\text { in the contralesional field }\end{array}$ & $\begin{array}{l}\text { No EEG or seizures mentioned, } \\
\text { "no history of previous neurolog- } \\
\text { ical or psychiatric disorders" }\end{array}$ & $\begin{array}{l}{[83]} \\
\text { Beniczky } \\
\text { et al. }\end{array}$ \\
\hline $\begin{array}{l}72 \text { Male } \\
\text { RH }\end{array}$ & $\begin{array}{l}\text { Right } \\
\text { occipital }\end{array}$ & Infarct & $\begin{array}{l}\text { Complex visual hallucinations* } \\
\text { in the contralesional field }\end{array}$ & $\begin{array}{l}\text { No EEG, seizures or psychiatric } \\
\text { comorbidity mentioned }\end{array}$ & $\begin{array}{l}{[84]} \\
\text { Cogan }\end{array}$ \\
\hline $\begin{array}{l}84 \text { Female } \\
\mathrm{H} \text { ? }\end{array}$ & $\begin{array}{l}\text { Right } \\
\text { occipital }\end{array}$ & Meningioma & $\begin{array}{l}\text { Complex visual hallucinations } \\
\text { in the contralesional visual field }\end{array}$ & $\begin{array}{l}\text { No EEG, seizures or psychiatric } \\
\text { comorbidity mentioned }\end{array}$ & $\begin{array}{l}{[85]} \\
\text { Nagaratnam } \\
\text { et al. }\end{array}$ \\
\hline 84 Female & $\begin{array}{l}\text { Right } \\
\text { occipital }\end{array}$ & Stroke & $\begin{array}{l}\text { Complex visual hallucinations } \\
\text { in the contralesional field }\end{array}$ & $\begin{array}{l}\text { No EEG, seizures or psychiatric } \\
\text { comorbidity mentioned, right } \\
\text { hypometabolism on SPECT }\end{array}$ & $\begin{array}{l}{[30]} \\
\text { Assadi } \\
\text { et al. }\end{array}$ \\
\hline $\begin{array}{l}63 \text { Male } \\
\text { RH }\end{array}$ & $\begin{array}{l}\text { Left } \\
\text { occipital }\end{array}$ & Infarct & $\begin{array}{l}\text { Complex visual hallucinations* } \\
\text { in the contralesional field }\end{array}$ & $\begin{array}{l}\text { No EEG, seizures or psychiatric } \\
\text { comorbidity mentioned }\end{array}$ & $\begin{array}{l}{[86]} \\
\text { Cole }\end{array}$ \\
\hline $\begin{array}{l}\text { ? Male } \\
\mathrm{H} \text { ? }\end{array}$ & $\begin{array}{l}\text { Left } \\
\text { occipital }\end{array}$ & Stroke & $\begin{array}{l}\text { Complex visual hallucinations* } \\
\text { in the contralesional visual field }\end{array}$ & $\begin{array}{l}\text { No EEG, seizures mentioned, } \\
\text { psychiatric tests were negative }\end{array}$ & $\begin{array}{l}{[87]} \\
\text { Anderson } \\
\text { \& Rizzo }\end{array}$ \\
\hline $\begin{array}{l}33 \text { Male } \\
\mathrm{H} \text { ? }\end{array}$ & $\begin{array}{l}\text { Left } \\
\text { occipital }\end{array}$ & $\begin{array}{l}\text { Arteriovenous } \\
\text { malfrmation }\end{array}$ & $\begin{array}{l}\text { Complex visual hallucinations } \\
\text { (palinoptic)* in the contralesi- } \\
\text { onal visual field }\end{array}$ & $\begin{array}{l}\text { No EEG, seizures or psychiatric } \\
\text { comorbidity mentioned }\end{array}$ & $\begin{array}{l}{[88]} \\
\text { Ritsema } \\
\text { \& Murphy }\end{array}$ \\
\hline $\begin{array}{l}70 \text { Male } \\
\mathrm{H} \text { ? }\end{array}$ & $\begin{array}{l}\text { Right } \\
\text { occipital }\end{array}$ & Infarct & $\begin{array}{l}\text { Complex hallucinations in } \\
\text { contralesional field }\end{array}$ & $\begin{array}{l}\text { No evidence of paroxysms in } \\
\text { EEG, no psychiatric comorbidity } \\
\text { reported }\end{array}$ & $\begin{array}{l}{[89]} \\
\text { Kompf } \\
\text { et al. }\end{array}$ \\
\hline $\begin{array}{l}50 \text { male } \\
\mathrm{H} \text { ? }\end{array}$ & $\begin{array}{l}\text { Right } \\
\text { occipital }\end{array}$ & Infarct & $\begin{array}{l}\text { Complex hallucinations } \\
\text { in contralesional field }\end{array}$ & $\begin{array}{l}\text { No evidence of paroxysms in } \\
\text { EEG, no psychiatric comorbidity } \\
\text { reported }\end{array}$ & $\begin{array}{l}{[89]} \\
\text { Kompf } \\
\text { et al. }\end{array}$ \\
\hline $\begin{array}{l}62 \text { Male } \\
\text { RH }\end{array}$ & $\begin{array}{l}\text { Left } \\
\text { occipital }\end{array}$ & Infarct & $\begin{array}{l}\text { Complex visual hallucinations, } \\
\text { including palinoptic* in the } \\
\text { contralesional field }\end{array}$ & $\begin{array}{l}\text { No evidence of paroxysms in } \\
\text { EEG, no psychiatric comorbidity } \\
\text { reported }\end{array}$ & $\begin{array}{l}{[90]} \\
\text { Huna-Baron } \\
\& \\
\text { Kupersmith }\end{array}$ \\
\hline $\begin{array}{l}54 \text { Male } \\
\mathrm{H} \text { ? }\end{array}$ & $\begin{array}{l}\text { Left } \\
\text { occipital }\end{array}$ & Infarct & $\begin{array}{l}\text { Complex visual hallucinations } \\
\text { in the ipsilesionalvisual field }\end{array}$ & $\begin{array}{l}\text { No EEG, seizures or psychiatric } \\
\text { comorbidity mentioned }\end{array}$ & $\begin{array}{l}{[91]} \\
\text { Manford } \\
\& \\
\text { Andermann }\end{array}$ \\
\hline $\begin{array}{l}79 \text { Male } \\
\text { RH }\end{array}$ & $\begin{array}{l}\text { Left } \\
\text { occipital }\end{array}$ & Infarct & $\begin{array}{l}\text { Complex visual hallucinations } \\
\text { in the ipsilesional visual field }\end{array}$ & $\begin{array}{l}\text { Normal EEG including during } \\
\text { hallucinations, "no personal or } \\
\text { family history of psychiatric } \\
\text { disease or substance abuse" }\end{array}$ & $\begin{array}{l}{[87]} \\
\text { Anderson } \\
\text { \& Rizzo }\end{array}$ \\
\hline $\begin{array}{l}82 \text { Female } \\
\text { RH }\end{array}$ & $\begin{array}{l}\text { Left } \\
\text { occipital }\end{array}$ & Infarct & $\begin{array}{l}\text { Simple visual hallucinations } \\
\text { (diplopia) in the contralesional } \\
\text { field }\end{array}$ & $\begin{array}{l}\text { No EEG, seizures or psychiatric } \\
\text { comorbidity reported }\end{array}$ & $\begin{array}{l}{[90] \text { Huna- }} \\
\text { Baron } \\
\& \\
\text { Kupersmith }\end{array}$ \\
\hline
\end{tabular}


Table 2, continued

\begin{tabular}{|c|c|c|c|c|c|}
\hline $\begin{array}{l}\text { Age at onset } \\
\text { gender and } \\
\text { hand preference }\end{array}$ & $\begin{array}{l}\text { Locus of } \\
\text { the lesion }\end{array}$ & Lesion etiology & $\begin{array}{l}\text { Type of hallucination and location } \\
\text { in the visual field(s) } \\
*=\text { the hallucinated image is from } \\
\text { episodic memory }\end{array}$ & Clinical considerations & Reference \\
\hline $\begin{array}{l}\text { 50 Male } \\
\text { RH }\end{array}$ & $\begin{array}{l}\text { Right } \\
\text { occipital }\end{array}$ & Thrombosis & $\begin{array}{l}\text { Simple hallucinations and complex } \\
\text { visual perseveretion (palinopsia) } \\
\text { of a few seconds in the contrale- } \\
\text { sional visual field }\end{array}$ & $\begin{array}{l}\text { No EEG, seizures or psychiatric } \\
\text { comorbidity mentioned }\end{array}$ & $\begin{array}{l}\text { [92] } \\
\text { Ardila } \\
\text { et al. }\end{array}$ \\
\hline $\begin{array}{l}64 \text { Female } \\
\mathrm{H} \text { ? }\end{array}$ & $\begin{array}{l}\text { Left } \\
\text { occipital }\end{array}$ & Infarct & $\begin{array}{l}\text { Simple hallucinations in contrale- } \\
\text { sional field }\end{array}$ & $\begin{array}{l}\text { Normal EEG with slowing over } \\
\text { the lesion, left occipital hypome- } \\
\text { tabolism on SPECT, no psychia- } \\
\text { tric comorbidity reported }\end{array}$ & $\begin{array}{l}{[31]} \\
\text { Flint } \\
\text { et al. }\end{array}$ \\
\hline $\begin{array}{l}37 \text { Female } \\
\mathrm{H} \text { ? }\end{array}$ & $\begin{array}{l}\text { Right } \\
\text { occipital }\end{array}$ & $\begin{array}{l}\text { Metastatic } \\
\text { carcinoma }\end{array}$ & $\begin{array}{l}\text { Simple hallucinations in the } \\
\text { contralesional field }\end{array}$ & $\begin{array}{l}\text { No EEG, seizures or psychiatric } \\
\text { comorbidity mentioned }\end{array}$ & $\begin{array}{l}{[93]} \\
\text { Johnson }\end{array}$ \\
\hline $\begin{array}{l}35 \text { Male } \\
\mathrm{H} \text { ? }\end{array}$ & $\begin{array}{l}\text { Left } \\
\text { occipital }\end{array}$ & Tuberculoma & $\begin{array}{l}\text { Simple visual hallucinations } \\
\text { (palinopsia) in the contralesional } \\
\text { visual field }\end{array}$ & $\begin{array}{l}\text { No EEG seizures or psychiatric } \\
\text { comorbidity mentioned }\end{array}$ & $\begin{array}{l}{[94]} \\
\text { Werring } \\
\text { \& Marsden }\end{array}$ \\
\hline $\begin{array}{l}84 \text { Female } \\
\mathrm{H} \text { ? }\end{array}$ & $\begin{array}{l}\text { Left } \\
\text { occipitl }\end{array}$ & Ischemia & $\begin{array}{l}\text { Simple long lasting hallucina- } \\
\text { tions in the contralesional field }\end{array}$ & $\begin{array}{l}\text { No EEG, seizures or psychiatric } \\
\text { comorbidity mentioned }\end{array}$ & $\begin{array}{l}\text { [24] Bosley } \\
\text { et al. }\end{array}$ \\
\hline $\begin{array}{l}\text { ? Female } \\
\mathrm{H} \text { ? }\end{array}$ & $\begin{array}{l}\text { Left } \\
\text { occipital }\end{array}$ & $\begin{array}{l}\text { Etiology not } \\
\text { specified }\end{array}$ & $\begin{array}{l}\text { Simple hallucinations in the } \\
\text { ipsilesional visual field }\end{array}$ & $\begin{array}{l}\text { No EEG seizures or psychiatric } \\
\text { comorbidity reported }\end{array}$ & $\begin{array}{l}\text { [87] Ander- } \\
\text { son } \\
\text { \& Rizzo }\end{array}$ \\
\hline $\begin{array}{l}22 \text { Male } \\
\mathrm{H} \text { ? }\end{array}$ & $\begin{array}{l}\text { Right } \\
\text { occipital }\end{array}$ & $\begin{array}{l}\text { Penetrating } \\
\text { wound }\end{array}$ & $\begin{array}{l}\text { Simple visual hallucinations } \\
\text { (polyopia) in the ipsilesional field }\end{array}$ & $\begin{array}{l}\text { No EEG seizures or psychiatric } \\
\text { comorbidity reported }\end{array}$ & $\begin{array}{l}{[75]} \\
\text { Bender }\end{array}$ \\
\hline
\end{tabular}

being able to describe and/or draw an object provided it is within visual reach. All the cases found had a left occipital lesion except the case of Riddoch [41] who had a left temporo-parietal lesion. As far as we could determine all these cases had lost visual evocation in both visual fields. See Table 4.

All 12 cases of Table 4 had a left hemisphere lesion. The two tailed binomial probability of this occurring by chance is $p<0.0005$. The profile strongly supports the psychic tonus model.

Most of the authors of these reports on loss of visual imagery are aware of the preponderance of left hemisphere lesions in loss of mental imagery. Most of them interpret their patient's loss as a verbal-imaginal problem. They believe language, particulary its semantic aspect, contributes a "code" which consolidates images in memory [42]. They seem to hint that there must exist a posterior module of language dedicated to this purpose or that modules of imagery are disconnected from those of language [12] -thus explaining why their patient is not aphasic outright. Several authors demonstrated that the patient could draw on copy but not from immediate memory [12,41,43-45]. Farah and colleagues [12] tested visual evocation likely to be totally unmediated verbally or semantically (pointing to the color corresponding to that of an object presented before) and indeed found a deficit. Several investigations found a deficit of facial recognition (recogniz- ing an unfamiliar face presented before) [12,46-48]. This particular mental operation is probably not mediated verbally or semantically. Several cases were not aphasic at all. Three cases complained of having stopped dreaming (the $[12,46,49]$ cases). Despite this, commentators still clung to a verbal (more specifically semantic) mediation concept of mental images to explain the lesion locus and deficit of visual evocation of the patients in Table 4. Our psychic tonus model proposes a different perspective on these cases. Without denying that language may certainly contribute to evocability of mental images our model proposes that there is simply a paucity of mental representation associated with activation of the intact right hemisphere and there is an exuberance of the same representation associated with activation of the intact left hemisphere. This balance of hemispheric specializations affects language as much as any other aspect of mental life, explaining why post lesion overtalkativeness (hyperlalia) is radically associated with right hemisphere lesions [3, 50]. Thus left hemisphere lesions can be expected to reduce spontaneous expression and evocability of both language and mental imagery as well as any other cognitive content (except perhaps for contents for which the right hemisphere is radically specialized, but we think there is no such thing).

Since visual hallucination and loss of visual imagery are two opposites, comparison of the 78 cases of oc- 
Table 3

Non-paroxystic visual hallucinations following a radiologically or surgically objectified unilateral occipital lobe lesion (location of the hallucinations in the visual fields unmentioned

\begin{tabular}{|c|c|c|c|c|c|}
\hline $\begin{array}{l}\text { Age at onset } \\
\text { gender and } \\
\text { hand preference }\end{array}$ & $\begin{array}{l}\text { Locus of } \\
\text { the lesion }\end{array}$ & $\begin{array}{l}\text { Lesion } \\
\text { etiology }\end{array}$ & Visual symptoms & Clinical considerations & Reference \\
\hline $\begin{array}{l}40 \text { Male } \\
\text { RH }\end{array}$ & $\begin{array}{l}\text { Left } \\
\text { occipito- } \\
\text { parietal }\end{array}$ & Infarct & $\begin{array}{l}\text { Complex visual hallucinations of } \\
\text { objects and persons (visual field } \\
\text { not specified) }\end{array}$ & $\begin{array}{l}\text { No EEG, no signs of seizures, } \\
\text { no psychiatric comorbidity } \\
\text { mentioned }\end{array}$ & $\begin{array}{l}\text { [95] } \\
\text { Bhaskaran } \\
\text { \& Prabhu }\end{array}$ \\
\hline $\begin{array}{l}82 \text { Male } \\
\mathrm{H} \text { ? }\end{array}$ & $\begin{array}{l}\text { Right } \\
\text { occipital }\end{array}$ & Infarct & $\begin{array}{l}\text { Complex visual hallucinations } \\
\text { (visual field not specified) }\end{array}$ & $\begin{array}{l}\text { No EEG, no signs of seizures, } \\
\text { no psychiatric comorbidity } \\
\text { mentioned }\end{array}$ & $\begin{array}{l}{[96]} \\
\text { Dejerine } \\
\text { et al. }\end{array}$ \\
\hline $\begin{array}{l}78 \text { Male } \\
\mathrm{H} \text { ? }\end{array}$ & $\begin{array}{l}\text { Left } \\
\text { occipital }\end{array}$ & Infarct & $\begin{array}{l}\text { Complex visual hallucinations } \\
\text { (visual field not specified) }\end{array}$ & $\begin{array}{l}\text { No EEG, no signs of seizures, } \\
\text { no psychiatric comorbidity } \\
\text { mentioned }\end{array}$ & $\begin{array}{l}{[96]} \\
\text { Dejerine } \\
\text { et al. }\end{array}$ \\
\hline $\begin{array}{l}56 \text { Male } \\
\mathrm{H} \text { ? }\end{array}$ & $\begin{array}{l}\text { Right } \\
\text { occipital }\end{array}$ & Infarct & $\begin{array}{l}\text { Complex visual hallucinations } \\
\text { (location unspecified) }\end{array}$ & $\begin{array}{l}\text { Normal EEG, no mention of } \\
\text { seizures, no psychiatric comor- } \\
\text { bidity reported }\end{array}$ & $\begin{array}{l}{[97]} \\
\text { Vaphiades } \\
\text { et al. }\end{array}$ \\
\hline $\begin{array}{l}46 \text { Male } \\
\mathrm{H} \text { ? }\end{array}$ & $\begin{array}{l}\text { Right } \\
\text { occipital }\end{array}$ & Infarct & $\begin{array}{l}\text { Complex visual hallucinations* } \\
\text { (location unspecified) }\end{array}$ & $\begin{array}{l}\text { Normal EEG, no mention of } \\
\text { seizures, no psychiatric comor- } \\
\text { bidity reported }\end{array}$ & $\begin{array}{l}{[97]} \\
\text { Vaphiades } \\
\text { et al. }\end{array}$ \\
\hline $\begin{array}{l}46 \text { Female } \\
\mathrm{H} \text { ? }\end{array}$ & $\begin{array}{l}\text { Right } \\
\text { occipital }\end{array}$ & Infarct & $\begin{array}{l}\text { Complex visual hallucinations } \\
\text { (location unspecified) }\end{array}$ & $\begin{array}{l}\text { Right slow focus in EEG, no } \\
\text { mention of seizures, no psy- } \\
\text { chiatric comorbidity reported }\end{array}$ & $\begin{array}{l}{[97]} \\
\text { Vaphiades } \\
\text { et al. }\end{array}$ \\
\hline $\begin{array}{l}72 \text { Male } \\
\mathrm{H} \text { ? }\end{array}$ & $\begin{array}{l}\text { Right } \\
\text { occipito- } \\
\text { temporal }\end{array}$ & Infarct & $\begin{array}{l}\text { Complex visual hallucinations } \\
\text { (location unspecified) }\end{array}$ & $\begin{array}{l}\text { EEG slowing on left side, no } \\
\text { mention of seizures, no psy- } \\
\text { chiatric comorbidity reported }\end{array}$ & $\begin{array}{l}{[97]} \\
\text { Vaphiades } \\
\text { et al. }\end{array}$ \\
\hline $\begin{array}{l}60 \text { Male } \\
\mathrm{H} ?\end{array}$ & $\begin{array}{l}\text { Right } \\
\text { occipital }\end{array}$ & Atheroma & $\begin{array}{l}\text { Complex visual hallucinations } \\
\text { (of objects and persons)* } \\
\text { location unspecified }\end{array}$ & $\begin{array}{l}\text { No EEG, seizures or psychiatric } \\
\text { comorbidity mentioned }\end{array}$ & $\begin{array}{l}{[93]} \\
\text { Johnson }\end{array}$ \\
\hline $\begin{array}{l}32 \text { Female } \\
\mathrm{H} \text { ? }\end{array}$ & $\begin{array}{l}\text { Right } \\
\text { parieto- } \\
\text { occiptal }\end{array}$ & Infarct & $\begin{array}{l}\text { Complex visual hallucinations* } \\
\text { (visual field not specified) }\end{array}$ & $\begin{array}{l}\text { No EEG, seizures or psychiatric } \\
\text { comorbidity mentioned }\end{array}$ & $\begin{array}{l}{[98]} \\
\text { Critchley }\end{array}$ \\
\hline $\begin{array}{l}67 \text { Male } \\
\text { RH }\end{array}$ & $\begin{array}{l}\text { Left } \\
\text { occipito- } \\
\text { temporal }\end{array}$ & Hemorrhage & $\begin{array}{l}\text { Complex visual hallucinations } \\
\text { (location unspecified) }\end{array}$ & $\begin{array}{l}\text { No EEG, seizures or psychiatric } \\
\text { comorbidity mentioned }\end{array}$ & $\begin{array}{l}{[99]} \\
\text { Ott } \\
\text { \& Saver }\end{array}$ \\
\hline $\begin{array}{l}66 \text { Female } \\
\mathrm{H} \text { ? }\end{array}$ & $\begin{array}{l}\text { Right } \\
\text { occipito- } \\
\text { parieto- } \\
\text { temporal }\end{array}$ & Hemorrhage & $\begin{array}{l}\text { Complex long lasting visual } \\
\text { hallucinations, prosopagnosia, } \\
\text { topographical agnosia }\end{array}$ & $\begin{array}{l}\text { No EEG, seizures or psychiatric } \\
\text { comorbidity mentioned }\end{array}$ & $\begin{array}{l}{[100]} \\
\text { Dutton }\end{array}$ \\
\hline $\begin{array}{l}71 \text { Female } \\
\mathrm{H} \text { ? }\end{array}$ & $\begin{array}{l}\text { Left } \\
\text { occipital }\end{array}$ & Infarct & $\begin{array}{l}\text { Complex visual hallucinations } \\
\text { (location unspecified) }\end{array}$ & $\begin{array}{l}\text { Normal EEG, no mention of } \\
\text { seizures, no psychiatric comor- } \\
\text { bidity reported }\end{array}$ & $\begin{array}{l}{[97]} \\
\text { Vaphiades } \\
\text { et al. }\end{array}$ \\
\hline $\begin{array}{l}67 \text { Female } \\
\mathrm{H} \text { ? }\end{array}$ & $\begin{array}{l}\text { Right } \\
\text { occipital }\end{array}$ & Infarct & $\begin{array}{l}\text { Complex visual hallucinations } \\
\text { (location unspecified) }\end{array}$ & $\begin{array}{l}\text { Normal EEG, no mention of } \\
\text { seizures, no psychiatric comor- } \\
\text { bidity reported }\end{array}$ & $\begin{array}{l}{[97]} \\
\text { Vaphiades } \\
\text { et al. }\end{array}$ \\
\hline $\begin{array}{l}62 \text { Male } \\
\mathrm{H} ?\end{array}$ & $\begin{array}{l}\text { Right } \\
\text { occipital }\end{array}$ & Infarct & $\begin{array}{l}\text { Simple visual hallucinations } \\
\text { (location unspecified) }\end{array}$ & $\begin{array}{l}\text { No EEG, seizures or psychiatric } \\
\text { comorbidity mentioned }\end{array}$ & $\begin{array}{l}\text { [97] } \\
\text { Vaphiades } \\
\text { et al. }\end{array}$ \\
\hline $\begin{array}{l}46 \text { Male } \\
\mathrm{H} \text { ? }\end{array}$ & $\begin{array}{l}\text { Right } \\
\text { occipito- } \\
\text { temporal }\end{array}$ & Infarct & $\begin{array}{l}\text { Palinopsia, not further } \\
\text { characterised (location } \\
\text { unspecified) }\end{array}$ & $\begin{array}{l}\text { No EEG, seizures or psychiatric } \\
\text { comorbidity mentioned }\end{array}$ & $\begin{array}{l}{[74]} \\
\text { Landis } \\
\text { et al. }\end{array}$ \\
\hline $\begin{array}{l}46 \text { Male } \\
\mathrm{H} \text { ? }\end{array}$ & $\begin{array}{l}\text { Right } \\
\text { occipito- } \\
\text { temporal }\end{array}$ & Infarct & $\begin{array}{l}\text { Visual hallucinations (not } \\
\text { further characterized nor } \\
\text { localized) }\end{array}$ & $\begin{array}{l}\text { No EEG, seizures or psychiatric } \\
\text { comorbidity mentioned }\end{array}$ & $\begin{array}{l}{[93]} \\
\text { Johnson }\end{array}$ \\
\hline
\end{tabular}


Table 3, continued

\begin{tabular}{|c|c|c|c|c|c|}
\hline $\begin{array}{l}\text { Age at onset } \\
\text { gender and } \\
\text { hand preference }\end{array}$ & $\begin{array}{l}\text { Locus of } \\
\text { the lesion }\end{array}$ & $\begin{array}{l}\text { Lesion } \\
\text { etiology }\end{array}$ & Visual symptoms & Clinical considerations & Reference \\
\hline $\begin{array}{l}35 \text { Male } \\
\mathrm{H} \text { ? }\end{array}$ & $\begin{array}{l}\text { Right } \\
\text { occipital }\end{array}$ & Ischemia & $\begin{array}{l}\text { Visual hallucinations (nature not fur- } \\
\text { ther specified, location unspecified) }\end{array}$ & $\begin{array}{l}\text { No EEG, seizures or psychiatric co- } \\
\text { morbidity mentioned }\end{array}$ & $\begin{array}{l}{[101]} \\
\text { Kanzaki } \\
\text { et al. }\end{array}$ \\
\hline $\begin{array}{l}42 \text { Male } \\
\mathrm{H} \text { ? }\end{array}$ & $\begin{array}{l}\text { Right } \\
\text { occipito- } \\
\text { parietal }\end{array}$ & $\begin{array}{l}\text { Cystic } \\
\text { lesion (unspeci- } \\
\text { fied etiology) }\end{array}$ & $\begin{array}{l}\text { Visual hallucinations palinopsia (lo- } \\
\text { cation unspecified) }\end{array}$ & $\begin{array}{l}\text { No mention of EEG, seizures or psy- } \\
\text { chiatric comorbidity }\end{array}$ & $\begin{array}{l}{[74]} \\
\text { Landis } \\
\text { et al. }\end{array}$ \\
\hline
\end{tabular}

Table 4

Non paroxystic bilateral loss of visual imagery in the waking state following a unilateral occipital lesion

\begin{tabular}{|c|c|c|c|c|c|}
\hline $\begin{array}{l}\text { Age at onset } \\
\text { gender and hand } \\
\text { preference }\end{array}$ & $\begin{array}{l}\text { Locus of } \\
\text { the lesion }\end{array}$ & $\begin{array}{l}\text { Lesion } \\
\text { etiology }\end{array}$ & Visual symptoms & Clinical considerations & Reference \\
\hline $\begin{array}{l}19 \text { Female } \\
\mathrm{H} \text { ? }\end{array}$ & $\begin{array}{l}\text { Left } \\
\text { occipital }\end{array}$ & $\begin{array}{l}\text { Closed head } \\
\text { injury }\end{array}$ & $\begin{array}{l}\text { Loss of mental images, associ- } \\
\text { ative agnosia, no aphasia }\end{array}$ & $\begin{array}{l}\text { No EEG paroxysms or psychia- } \\
\text { tric comorbidity mentioned }\end{array}$ & $\begin{array}{l}\text { [48] } \\
\text { Davidoff } \\
\text { \& Wilson }\end{array}$ \\
\hline $\begin{array}{l}51 \text { Male } \\
\mathrm{H} \text { ? }\end{array}$ & $\begin{array}{l}\text { Left } \\
\text { occipital }\end{array}$ & $\begin{array}{l}\text { Closed head } \\
\text { injury }\end{array}$ & $\begin{array}{l}\text { Loss of mental images, seman- } \\
\text { tic access agnosia, no aphasia }\end{array}$ & $\begin{array}{l}\text { No EEG, paroxysms or psychia- } \\
\text { tric comorbidity mentioned }\end{array}$ & $\begin{array}{l}{[45]} \\
\text { Riddoch } \\
\& \\
\text { Humphreys }\end{array}$ \\
\hline $\begin{array}{l}56 \text { Male } \\
\text { RH }\end{array}$ & $\begin{array}{l}\text { Left } \\
\text { temporo- } \\
\text { occipital }\end{array}$ & Infarct & $\begin{array}{l}\text { Loss of mental images, visuo- } \\
\text { imaginal constructional apraxia, } \\
\text { no aphasia }\end{array}$ & $\begin{array}{l}\text { No EEG, paroxysms or psychia- } \\
\text { tric comorbidity mentioned }\end{array}$ & $\begin{array}{l}{[42]} \\
\text { Grossi } \\
\text { et al. }\end{array}$ \\
\hline $\begin{array}{l}60 \text { Male } \\
\mathrm{H} \text { ? }\end{array}$ & $\begin{array}{l}\text { Left } \\
\text { occipital }\end{array}$ & Infarct & $\begin{array}{l}\text { Loss of mental images, optic } \\
\text { aphasia (partial loss of verbal } \\
\text { account of mental images), } \\
\text { aphasia limited to visual } \\
\text { mediation }\end{array}$ & $\begin{array}{l}\text { No EEG, paroxysms or psychia- } \\
\text { tric comorbidity mentioned }\end{array}$ & $\begin{array}{l}{[102]} \\
\text { Manning } \\
\text { \& Campbell }\end{array}$ \\
\hline $\begin{array}{l}83 \text { Male } \\
\text { RH }\end{array}$ & $\begin{array}{l}\text { Left } \\
\text { temporo- } \\
\text { occipital }\end{array}$ & $\begin{array}{l}\text { Infarct and } \\
\text { hemorrhage }\end{array}$ & $\begin{array}{l}\text { Loss of visual imagery, mild } \\
\text { anomia without other signs of } \\
\text { aphasia }\end{array}$ & $\begin{array}{l}\text { No EEG, paroxysms or psychia- } \\
\text { tric comorbidity mentioned }\end{array}$ & $\begin{array}{l}{[44]} \\
\text { Goldenberg }\end{array}$ \\
\hline $\begin{array}{l}65 \text { Male } \\
\text { RH }\end{array}$ & $\begin{array}{l}\text { Left } \\
\text { occipito- } \\
\text { parietal }\end{array}$ & Infarct & $\begin{array}{l}\text { Loss of visual imagery, agraphia, } \\
\text { Gerstmann syndrome, word } \\
\text { finding difficulty }\end{array}$ & $\begin{array}{l}\text { No EEG, paroxysms or psychia- } \\
\text { tric comorbidity mentioned }\end{array}$ & $\begin{array}{l}{[103]} \\
\text { Levine } \\
\text { et al. }\end{array}$ \\
\hline $\begin{array}{l}79 \text { Male } \\
\mathrm{H} \text { ? }\end{array}$ & $\begin{array}{l}\text { Left } \\
\text { occipito- } \\
\text { parietal }\end{array}$ & Infarct & $\begin{array}{l}\text { Loss of visual imagery, dyslexia, } \\
\text { anomia, ipsilesional neglect }\end{array}$ & $\begin{array}{l}\text { No EEG, paroxysms or psychia- } \\
\text { tric comorbidity mentioned }\end{array}$ & $\begin{array}{l}{[104]} \\
\text { Cocchini } \\
\text { et al. }\end{array}$ \\
\hline $\begin{array}{l}63 \text { Male } \\
\text { RH }\end{array}$ & $\begin{array}{l}\text { Left } \\
\text { temporo- } \\
\text { occipital }\end{array}$ & Infarct & $\begin{array}{l}\text { Loss of visual imagery, alexia } \\
\text { and anomia for visually } \\
\text { presented objects were the only } \\
\text { aphasic signs, loss of dreaming }\end{array}$ & $\begin{array}{l}\text { No EEG, paroxysms or psychia- } \\
\text { tric comorbidity mentioned }\end{array}$ & $\begin{array}{l}{[46]} \\
\text { Basso } \\
\text { et al. }\end{array}$ \\
\hline $\begin{array}{l}64 \text { Male } \\
\text { RH }\end{array}$ & $\begin{array}{l}\text { Left } \\
\text { temporo- } \\
\text { occipital }\end{array}$ & Infarct & $\begin{array}{l}\text { Loss of visual imagery, no } \\
\text { aphasia, loss of dreaming }\end{array}$ & $\begin{array}{l}\text { EEG "mildly abnormal", no } \\
\text { paroxysms or psychiatric } \\
\text { comorbidity mentioned }\end{array}$ & $\begin{array}{l}{[12]} \\
\text { Farah } \\
\text { et al. }\end{array}$ \\
\hline $\begin{array}{l}47 \text { Male } \\
\text { RH }\end{array}$ & $\begin{array}{l}\text { Left } \\
\text { temporo- } \\
\text { occipital }\end{array}$ & Infarct & $\begin{array}{l}\text { Loss of mental images, optic } \\
\text { aphasia, loss of dreaming, no } \\
\text { aphasia }\end{array}$ & $\begin{array}{l}\text { No EEG, paroxysms or psychia- } \\
\text { tric comorbidity mentioned }\end{array}$ & $\begin{array}{l}{[49]} \\
\text { Pena- } \\
\text { Casanova } \\
\text { et al. }\end{array}$ \\
\hline $\begin{array}{l}35 \text { Male } \\
\text { RH }\end{array}$ & $\begin{array}{l}\text { Left } \\
\text { temporo- } \\
\text { parieto- } \\
\text { occipital }\end{array}$ & $\begin{array}{l}\text { Resected } \\
\text { meningioma }\end{array}$ & $\begin{array}{l}\text { Loss of visual imagery, } \\
\text { dysphasia }\end{array}$ & $\begin{array}{l}\text { No EEG, paroxysms or psychia- } \\
\text { tric comorbidity mentioned }\end{array}$ & $\begin{array}{l}{[43]} \\
\text { Deleval } \\
\text { et al. }\end{array}$ \\
\hline $\begin{array}{l}43 \text { Female } \\
\text { RH }\end{array}$ & $\begin{array}{l}\text { Left } \\
\text { parieto- } \\
\text { occipital }\end{array}$ & Hemorrhage & $\begin{array}{l}\text { Loss of mental images, visual } \\
\text { associative agnosia, evolving } \\
\text { to optic agnosia }\end{array}$ & $\begin{array}{l}\text { No EEG, paroxysms or psychia- } \\
\text { tric comorbidity mentioned }\end{array}$ & $\begin{array}{l}{[47]} \\
\text { Benke }\end{array}$ \\
\hline
\end{tabular}


cipital post lesion visual hallucination (all the cases of Table 1) with the 12 cases of occipital post lesion loss of visual imagery (all the cases of Table 4) - for lesion side appears perfectly meaningful. The double crossed dissociation predicted by the psychic tonus model is highly significant $\left(\mathrm{Chi}^{2}=15.8 p<0.0005\right)$.

\subsection{Secondary control analyses (all the cases $N=$ 90)}

At first glance the results reported above seem to be in total concordance with the hypothesis of the psychic tonus model. Furthermore, the stronger lateralization of the hypognosic group than the hypergnosic group is also in accord with the literature reviewed in the introduction. However several contaminating variables could challenge our main result.

\subsection{The locus of the lesion}

Visual hallucinations (including palinopsia) and the loss of mental imagery are more often the result of an occipital or temporal lobe lesion than lesions located elsewhere (see [51] for a review). In the current study, occipital damage was an inclusion criterion but damage elsewhere was not an exclusion criterion. It was therefore thought useful to verify whether a lobar difference in lesion extension could explain the main finding of a crossed interaction between lesion side and type of visual disorder (hypo/hypergnosia). The locus of the lesion was coded for each lobe (frontal, temporal, or parietal) separately because a combined analysis would have violated the rule of independence of the replicates and cases. We partialed out presence/absence of a lesion in each lobe as well as volume of the lesion (number of lobes lesioned) in partial correlation analysis between lesion side and type of visual disturbance. The correlation (interaction) remained significant in each case $(\operatorname{Rp}>0.41, p<0.0005)$.

\subsection{Presence of aphasic symptoms}

Presence of aphasic symptoms (anomia, dysphasia, aphasia, impaired oral comprehension) was of interest because of the well documented hemispheric specialization of the left hemisphere for language (see [52] for a review). Presence/absence of aphasic symptoms could compete with the psychic tonus model. Such an eventuality would require a caveat in our interpretation of our main findings, or at the very least, statistical partialing out of this variable's effect in our test of the main hypothesis. As we expected their were more cases with aphasic symptoms in the hypognosic group $(5 / 12)$ than in the hypergnosic group $(1 / 78)\left(\mathrm{Chi}^{2}=\right.$ 27.3, $p<0.0005)$. However the crossed interaction between lesion side and type of visual disturbance remained robust after partialing out of presence/absence of aphasia $(\mathrm{Rp}>0.38, p<0.0005)$.

Several other variables were also tested for the eventuality of providing an alternative explanation for the finding of a crossed dissociation of left and right lesioned cases as a function of type of visual disturbance. For example psychiatric comorbidity (psychosis or mania) could be associated with visual hallucination [53] and not loss of imaginal evocation. A sampling bias of etiology of the lesion is important to consider because irritative pathophysiology (ex: tumor) could cause effects contrary to stable lesions such as CVAs. Another concern is that certain etiologies (ex: head trauma) comprise subtle diffuse damage thereby compromizing the "lesion side" inference. A sampling bias of hand writing preference would be important because a significant proportion of left handers have inverted hemispheric specialization (at least for language). Age is of concern because juveniles are less hemispherically specialized than adults and because the very elderly have diffuse brain atrophy and could thus present "noisy" lesion side characterization as well. A gender sampling bias is relevant to consider because in many respects women have less hemispheric specialization than men [54]. Date of publication is pertinent because diagnostic methods have improved over the decades, particularly due to the passage from CT to MRI scanning. However, the crossed interaction between lesion side and type of visual disturbance remained robust after partialing out of all these variables $(\mathrm{Rp}>0.40, p<$ 0.0005). In fact, none the control variables mentioned above, was significantly related to the interaction between lesion side and type of visual disturbance.

\subsection{More specific secondary control analyses (hallucination cases only $N=78$ )}

\subsection{Perilesional release}

Occipitally lesioned cases often present with hemianopia. In the neurologic literature it is well known that visual hallucinations often occur in the defective visual field [55]. It is thus of interest to analyse the incidence of hallucinations in each visual field. Of the 39 cases with hallucinations in a single field, 36 hallucinated in the contralesional field and only 3 in the 
ipsilesional field $\left(\mathrm{Chi}^{2}=27.9, p<0.0005\right)$ indicating strong (though indirect) support for the idea according to which the source of the hallucination is perilesional (ipsilesional) release. Bosley and colleagues [24] provide a particularly interesting description of perilesional hyperperfusion during unilateral visual hallucination: they illustrate a slim ring of hyperperfusion on SPECT all around the lesion. It is noteworthy that unilateral hallucination is nearly always contralesional whether the lesion be in the left or right occipital lobe.

\subsection{Contralesional release}

Perilesional release, we think, could be independent of hemispheric specialization. After all, it is observed as a highly localized phenomenon-close to the lesion, and it probably generates only unilateral hallucinations (although that remains to be determined). The psychic tonus model does not contest perilesional release. Rather, it proposes that there should be more hallucinating cases with right hemisphere lesions and it supposes that that preponderance would be due to contralesional release. What then, in the current data base would be the evidence for contralesional release? First, with regard to EEG, there are 12 cases out of the grand ensemble of 90 manifesting unilateral EEG slowing, and ten of the twelve cases had ipsilesional slowing. This is a profile compatible with contralesional release. In addition, in the cases with EEG $(\mathrm{N}=24$, overall), in whom we are more certain that the source of hallucination was not perilesional paroxystic irritation, there was a significant preponderance of right hemisphere cases $\left(\mathrm{Chi}^{2}=6.0, p<0.015\right)$. Secondly, in the grand ensemble of 90 cases here, there are six cases with unilateral hypoperfusion on SPECT, all ipsilesional. This is also compatible with contralesional release. Of these six cases, four $(67 \%)$ had a right hemisphere lesion (NS).

We propose that it would be worthwhile to further investigate both eventualities, of perilesional hypermetabolism and of ipsilesional hypometabolism, as a function of presence or absence of hallucination, in one or both visual fields. We expect it will be found that the two types of mediation of hallucination occur, and even that each can occur in a same patient. We also expect that perilesional hypermetabolism will be found to be more locally distributed (around the lesion) and that ipsilesional hypometabolism will be found to be more widespread, diffusing throughout most of the hemisphere.

\subsection{Complexity of the hallucination}

A visual hallucination can be either simple (seeing colored spots flickering lights, etc., $\mathrm{N}=13 / 78$ cases here) or complex (seeing a living scene, an animal, a person etc., $\mathrm{N}=62 / 78$ cases here). The complexity of hallucination, thus defined, was not significantly associated with lesion side. Whether the hallucinations occurred throughout both visual fields $(\mathrm{N}=21 / 78)$ or were restricted to only one field $(\mathrm{N}=39 / 78)$ was unrelated to lesion side. Finally, cases whose hallucination(s) included elements of episodic memory (elements highly specific to the patient's history) $(\mathrm{N}=$ 28/78) were not more likely to have right hemisphere lesions than the cases without episodic memory content $(\mathrm{N}=47 / 78)$.

\section{Discussion}

The results of this investigation support the psychic tonus model. Unilateral occipital lesions causing a "positive" disturbance of visual representation (hypergnosia) are more often located in the right hemisphere. Unilateral occipital lesions causing "negative" disturbance of visual representation (hypognosia) are always located in the left hemisphere of right handers.

Why would a focal left occipital lesion impair a patient's visual representation of the entire visual field? Such an effect cannot be attributed to damage of primary sensory tracts. Primary visual representation has been mapped in many species and has always been found to be strictly contralateral and to present the same topography in each hemisphere in each occipital lobe, including in man [56]. Likewise, why would a right hemisphere lesion be more likely to provoke hallucinations in both visual fields? These are high order cognitive phenomena not explainable as direct neurosensory effects. We propose that the cerebral hemispheres influence higher level representation via psychic tonus regardless of the modality (motor, somatic, auditory, visual, immune, etc.). Psychic tonus may be only one among several sorts of hemispheric specialization, or it may eventually be made to fit into a more general and well integrated theory of hemispheric specialization. Hemispheric specialization for psychic tonus is a proposition that goes against the dominant neuroscience zeitgeist of today which is cognitivism. Psychic tonus is an unusual way of contemplating telencephalic and a fortiori cortical function. Unconscious and/or involuntary functions have more typical- 
ly been conceived to depend on phylogenetically primitive brain tissue (e.g. the autonomic nervous system, the limbic system), and that might indeed be the case, but the lesion data indicate so far that psychic tonus is neocortically implemented in the human brain.

It could be thought that the main mediator of the post lesion "sticky switch" is one or several neurotransmitters. Three candidates are central norepinephrine, serotonin and dopamine (all known to modulate functions subsumed under psychic tonus). Robinson and colleagues [57,58] ligated the middle cerebral artery of rats. Assays of brain catecholamines revealed 30 percent reductions of norepinephrine in the injured and uninjured cortex and locus coeruleus and a 20 percent reduction of dopamine in the substantia nigra in the right lesioned rats who had also become hyperactive. In contrast rats with left middle cerebral artery ligations did not become hyperactive and did not show any significant change in catecholamines in any of the brain areas studied. Similar asymmetry occurs in serotonin concentrations as a function of stroke side in humans [59]. It remains to be determined whether the latter effect is hemispherically symmetrical or not (e.g., a PET ligand study). In fact however we believe that such a neurotransmitter-mediated mechanism is not the principal determinant of hemispheric specialization for psychic tonus effects reported here and elsewhere. Superficial cuts in rat cortex modulate psychic tonus without affecting neurotransmitter concentrations [1], and ictal visual hallucination results far more often from left foci [60]. This corroboration of hemispheric specialization for psychic tonus cannot be explained as a chronic and major change of neurotransmitter concentration in the brain after lesions. In other words, it is presently best explained as true hemispheric specialization, in the full sense.

\section{Acknowledgments}

This research was made possible by a grant from the Fonds de Recherche en Santé du Québec (FRSQ).

\section{References}

[1] C.M.J. Braun, Evolution of hemispheric specialisation of antagonistic systems of management of the body's energy, Laterality 12 (2007), 397-427.

[2] C.M.J. Braun, C. Larocque, S. Daigneault and I. MontourProulx, Mania, pseudomania, depression, and pseudodepression resulting from focal unilateral cortical lesions, $\mathrm{Neu}$ ropsychiatry Neuropsychol Behav Neurol 12 (1999), 35-51.
[3] C.M.J. Braun, R. Daigneault and A. Guimond, DSM-IV symptoms of mania: Which one(s) result more often from right than left hemisphere lesions? Compr Psychiatry (2008) in press.

[4] C.M.J. Braun, M. Dumont, J. Duval, I. Hamel and L. Godbout, Opposed left and right brain hemisphere contributions to sexual drive: a multiple lesion case analysis, Behav Neurol 14 (2003), 55-61.

[5] C.M.J. Braun, M. Dumont, J. Duval and I. Hamel-Hebert, Speech rate as a sticky switch: a multiple lesion case analysis of mutism and hyperlalia, Brain Lang 89 (2004), 243-252.

[6] A. St-Marseille, G. Kouassi, P. D'Angelo, S. Laplante, M.L. Cheng, F. Trouvé, D. Gilbert, M. Geadah and C.M.J. Braun, Opposed left and right neocortical involvement in the immune response indexed by cerebrovascular accidents in humans: preliminary results, Brain Cogn 32 (1996), 173175.

[7] C.M.J. Braun, J. Duval and A. Guimond, Auditory hypergnosia as an example of psychic tonus in the temporal lobes: multiple case analyses, Crit Rev Neurobiol 17 (2005), 145160.

[8] C.M.J. Braun, S. Desjardins, S. Gaudelet and A. Guimond, Psychic tonus, body schema and parietal lesions: A multiple lesion case analysis, Behav Neurol 18 (2007), 1-16.

[9] C.M.J. Braun, J. Delisle, I. Rouleau, A. Guimond and R. Daigneault, Post lesion dysfunctional hyper and hypomnesia: Evidence for opposed hemispheric specialization, Laterality, 2008, in press.

[10] E.F. Bazhin, Y.A. Meerson and I.M. Tonkonogii, On distinguishing a visual signal from noise by patients with visual agnosia and visual hallucinations, Neuropsychologia 11 (1973), 319-324.

[11] R. Cabeza and L. Nyberg, Imaging Cognition II: An Empirical Review of 275 PET and fMRI Studies, J Cogn Neurosci 12 (2000), 1-47.

[12] M.J. Farah, D.N. Levine and R. Calvanio, A case study of mental imagery deficit, Brain Cogn 8 (1988), 147-164.

[13] G. Goldenberg and C. Artner, Visual imagery and knowledge about the visual appearance of objects in patients with posterior cerebral artery lesions, Brain Cogn 15 (1991), 160-186.

[14] D.C. Gillepsie, A. Bowen and J.K. Foster, Memory impairment following right hemisphere stroke: a comparative metaanalytic and narrative review, Clin Neuropsychol 20 (2006), 59-75.

[15] N. Oishi, F. Udaka, M. Kameyama, N. Sawamoto, K. Hashikawa and H. Fukuyama, Regional cerebral blood flow in Parkinson disease with nonpsychotic visual hallucinations, Neurology 65 (2005), 1708-1715.

[16] A. Nagano-Saito, Y. Washimi, Y. Arahata, K. Iwai, S. Kawatsu, K. Ito, A. Nakamura, Y. Abe, T. Yamada, T. Kato and T. Kachi, Visual hallucination in Parkinson's disease with FDG PET, Mov Disord 19 (2004), 801-806.

[17] J. O’Brien, M. Firbank, U. Mosimann, D. Burn and I. McKeith, Change in perfusion, hallucinations and fluctuations in consciousness in dementia with Lewy bodies, Psychiatry Res 139 (2005), 79-88.

[18] T. Imamura, K. Ishii, N. Hirono, M. Hashimoto, S. Tanimukai, H. Kazuai, T. Hanihara, M. Sasaki and E. Mori, Visual hallucinations and regional cerebral metabolism in dementia with Lewy bodies (DLB), Neuroreport 10 (1999), 1903-1907.

[19] T. Mori, M. Ikeda, R. Fukuhara, P.J. Nestor and H. Tanabe, Correlation of visual hallucinations with occipital rCBF 
changes by donepezil in DLB, Neurology 66 (2006), 935937.

[20] N. Adachi, T. Watanabe, H. Matsuda and T. Onuma, Hyperperfusion in the lateral temporal cortex, the striatum and the thalamus during complex visual hallucinations: Single photon emission computed tomography findings in patients with Charles Bonnet Syndrome, Psychiatry Clin Neurosci 54 (2000), 157-162.

[21] J. Shedlack, W.M. McDonald, D.T. Laskowitz and K.R. Krishnan, Geniculocalcarine hyperintensities on brain magnetic resonance imaging associated with visual hallucinations in the elderly, Psychiatry Res 54 (1994), 283-293.

[22] C.M.J. Braun, M. Dumont, J. Duval, I. Hamel-Hebert and L. Godbout, Brain modules of hallucination: an analysis of multiple patients with brain lesions, $J$ Psychiatry Neurosci 28 (2003), 432-449.

[23] C. Lefebre and H.W. Kolmel, Palinopsia as an epileptic phenomenon, Eur Neurol 29 (1989), 323-327.

[24] T.M. Bosley, R. Dann, F.L. Silver, A. Alavi, M. Kushner, J.B. Chawluk, P.J. Savino, R.C. Sergott, N.J. Schatz and M. Reivich, Recovery of vision after ischemic lesions: Positron emission tomography, Annals of Neurology 21 (1987), 444450.

[25] R. Hayashi, S. Shimizu, R. Watanabe, Y. Katsumata and M. Mimura, Palinopsia and perilesional hyperperfusion following subcortical hemorrhage, Acta Neurol Scand 105 (2002), 228-231.

[26] G.C. Vogeley, Pictorial pseudohallucinations with an "aperture effect" in a patient with quadrantanopia, $\mathrm{J}$ Neurol Neurosurg Psychiatry 65 (1998), 275-277.

[27] H. Tanaka, T. Takagi, A. Takeda and T. Hamanaka, Palinopsia in the center of right visual field associated with left occipital lobe lesion, Japanese Journal of Neuropsychology 15 (1999), $181-186$.

[28] K. Machii, Y. Watanabe, Y. Narita, S. Kuzuhara and T. Meza$\mathrm{ki}$, [123I-IMP SPECT findings of visual perseveration in a patient with old hemorrhage in the right occipital lobe] [Article in Japanese], Rinsho Shinkeigaku 34 (1994), 823-826.

[29] M. Satoh, K. Suzuki, M. Miyamura, R. Katoh and S. Kuzuhara, [Metamorphopsia and transient increase in the cerebral blood flow of the left occipital pole on 123IIMP SPECT: a case report] [Article in Japanese], Rinsho Shinkeigaku 37 (1997), 631-635.

[30] M. Assadi, S. Baseman and D. Hyman, Tc SPECT scan in a patient with occipital lobe infarction and complex visual hallucinations, J Neurosci Nurs 35 (2003), 175-177.

[31] A.C. Flint, J.P. Loh and J.C. Brust, Vivid visual hallucinations from occipital lobe infarction, Neurology 65 (2005), 756.

[32] I. Hoksbergen, B.A. Pickut, P. Marien, H. Slabbynck, J. Kunnen and P.P. De Deyn, SPECT findings in an unusual case of visual hallucinosis, J Neurol 243 (1996), 594-598.

[33] Y. Ii, T. Sakai and S. Kuzuhara, [Complex visual hallucination in the quadrantanopsic field in a patient with a medial temporal hematoma following left putaminal hemorrhage] [Article in Japanese], Rinsho Shinkeigaku 40 (2000), 19-23.

[34] T. Kishi, J. Uegaki, M. Kitani, A. Fujimoto and R. Naganuma, The usefulness of single photon emission computed tomography in Charles Bonnet syndrome: a case with occipital lobe involvement, Gen Hosp Psychiatry 22 (2000), 132-135.

[35] Y.T. Sun and C.C. Lin, Sequential appearance and disappearance of hemianopia, palinopsia and metamorphopsia: a case report and literature review, Acta Neurol Taiwan 13 (2004), 77-83.
[36] E.J. Choi, J.K. Lee, J.K. Kang and S.A. Lee, Complex visual hallucinations after occipital cortical resection in a patient with epilepsy due to cortical dysplasia, Arch Neurol 62 (2005), 481-484.

[37] T. Inafuku, F. Sakai, T. Sakamoto, T. Iizuka and T. Kanda, [Visual hallucination in the hemianopic field caused by dural arteriovenous malformation], Rinsho Shinkeigaku 34 (1994), 484-488.

[38] M.L. Paillère-Martinot, M.H. Dao-Castellana, M.C. Masure, B. Pillon and J.L. Martinot, Delusional misidentification: a clinical, neuropsychological and brain imaging case study, Psychopathology 27 (1994), 200-210.

[39] H.W. Kolmel, Complex visual hallucinations in the hemianopic field, J Neurol Neurosurg Psychiatry 48 (1985), 2938.

[40] J. Duval, C.M.J. Braun, I. Montour-Proulx, S. Daigneault, I. Rouleau and J. Bégin, Brain lesions and IQ: Recovery versus decline depends on age of onset, J Child Adolesc Neurol 20 (2008), 1-12.

[41] M.J. Riddoch, Loss of visual imagery: A generation deficit, Cogn Neuropsychol 7 (1990), 249-273.

[42] D. Grossi, A. Orsini, A. Modafferi and M. Liotti, Visuoimaginal constructional apraxia: on a case of selective deficit of imagery, Brain Cogn 5 (1986), 255-267.

[43] J. Deleval, J. De Mol and J. Noterman, [Loss of mental images], Acta Neurol Belg 83 (1983), 61-79.

[44] G. Goldenberg, Loss of visual imagery and loss of visual knowledge - a case study, Neuropsychologia 30 (1992), 1081-1099.

[45] M.J. Riddoch and G.W. Humphreys, A case of integrative visual agnosia, Brain 110 (Pt 6) (1987), 1431-1462.

[46] A. Basso, E. Bisiach and C. Luzzatti, Loss of mental imagery: a case study, Neuropsychologia 18 (1980), 435-442.

[47] T. Benke, Visual agnosia and amnesia from a left unilateral lesion, Eur Neurol 28 (1988), 236-239.

[48] J. Davidoff and B. Wilson, A case of visual agnosia showing a disorder of pre-semantic visual classification, Cortex $\mathbf{2 1}$ (1985), 121-134.

[49] J. Pena-Casanova, T. Roig-Rovira, A. Bermudez and E. Tolosa-Sarro, Optic aphasia, optic apraxia, and loss of dreaming, Brain Lang 26 (1985), 63-71.

[50] C.M.J. Braun, M. Dumont, J. Duval, I. Hamel and L. Godbout, Opposed left and right brain hemisphere contributions to sexual drive: A multiple lesion case analysis, Behav $\mathrm{Neu}$ rol 14 (2002), 55-61.

[51] H.W. Kolmel, Visual illusions and hallucinations, Baillieres Clin Neurol 2 (1993), 243-264.

[52] J. Hutsler and R.A. Galuske, Hemispheric asymmetries in cerebral cortical networks, Trends Neurosci 26 (2003), 429435.

[53] J.W. Norton and J.J. Corbett, Visual perceptual abnormalities: hallucinations and illusions, Semin Neurol 20 (2000), $111-121$.

[54] J.W. Miller, S. Jayadev, C.B. Dodrill and G.A. Ojemann, Gender differences in handedness and speech lateralization related to early neurologic insults, Neurology 65 (2005), 1974-1975.

[55] E. Vanroose, M. Marchau, I. Dehaene and M. Lammens, Altitudinal hemianopia; a clinical and anatomical entity or a mere coincidence? Case report and review of literature, Acta Neurol Belg 90 (1990), 254-264.

[56] I. Klein, J. Dubois, J.F. Mangin, F. Kherif, G. Flandin, J.B. Poline, M. Denis, S.M. Kosslyn and D. Le Bihan, Retinotopic organization of visual mental images as revealed by func- 
tional magnetic resonance imaging, Brain Res Cogn Brain Res 22 (2004), 26-31.

[57] R.G. Robinson, Differential behavioral and biochemical effects of right and left hemispheric cerebral infarction in the rat, Science 205 (1979), 707-710.

[58] R.G. Robinson and J.T. Coyle, Lateralization of catecholaminergic and behavioral response to cerebral infarction in the rat, Life Sci 24 (1979), 943-950.

[59] R.G. Robinson and S.E. Starkstein, Mood disorders following stroke: new findings and future directions, $J$ Geriatr Psychiatry 22 (1989), 1-15.

[60] A. Guimond and C.M.J. Braun, Hemispheric specialization revealed by ictal halluciation: Findings from 217 cases with a unilateral epileptic focus. Epilept Beh (submitted 2008).

[61] E.M. Michel and B.T. Troost, Palinopsia: cerebral localization with computed tomography, Neurology 30 (1980), 887889.

[62] J.C. Meadows and S.S. Munro, Palinopsia, J Neurol Neurosurg Psychiatry 40 (1977), 5-8.

[63] J.C. La Mancusa and A.R. Cole, Visual manifestations of occipital lobe infarction in three patients on a geriatric psychiatry unit, J Geriatr Psychiatry Neurol 1 (1988), 231-234.

[64] J.W. Lance, Simple formed hallucinations confined to the area of a specific visual field defect, Brain 99 (1976), 719 734.

[65] S.J. Peroutka, B.H. Sohmer, A.J. Kumar, M. Folstein and R.G. Robinson, Hallucinations and delusions following a right temporoparietooccipital infarction, Johns Hopkins Med J 151 (1982), 181-185.

[66] J. Dodd, A. Heffeman and J. Blake, Visual hallucinations associated with Charles Bonnet syndrome an ever increasing diagnosis, Ir Med J 92 (1999), 344-345.

[67] J.L. Medina, S. Chokroverty and F.A. Rubino, Syndrome of agitated delirium and visual impairment: a manifestation of medial temporo-occipital infarction, J Neurol Neurosurg Psychiatry 40 (1977), 861-864.

[68] P. Lunardi, L. Tacconi, P. Missori and M. Salvati, Palinopsia: unusual presenting symptom of a cerebral abscess in a man with Kartagener's syndrome, Clin Neurol Neurosurg 93 (1991), 337-339.

[69] M.C. Patterson, I.H. Bunce and M.J. Eadie, Cerebral abscess in leukaemia: an unusual presentation of a rare complication, Clin Exp Neurol 21 (1985), 257-262.

[70] F. Maillot, C. Belin, D. Perrier and P. Larmande, [Visual perseveration and palinopsia: a visual memory disorder?], Rev Neurol (Paris) 149 (1993), 794-796.

[71] E.B. Mukaetova-Ladinsk, C. Dibben, L. Allen, S. Ferrera and J. Darley, Temporary visual hallucinations. A case report of complex visual hallucinations due to right occipital lobe haematoma, CPT Bulletin of old Age Psychiatry 3 (2002), $51-53$.

[72] S. Strandgaard, G.S. Andersen, P. Ahlgreen and P.E. Nielsen, Visual disturbances and occipital brain infarct following acute, transient hypotension in hypertensive patients, Acta Med Scand 216 (1984), 417-422.

[73] R.P. Lazaro, Palinopsia: rare but ominous symptom of cerebral dysfunction, Neurosurgery 13 (1983), 310-313.

[74] T. Landis, J.L. Cummings, L. Christen, J.E. Bogen and H.G. Imhof, Are unilateral right posterior cerebral lesions sufficient to cause prosopagnosia? Clinical and radiological findings in six additional patients, Cortex 22 (1986), 243-252.

[75] M.B Bender, Polyopia and monocular diplopia of cerebral origin, Arch Neurol Psychiatry 54 (1945), 323-338.
[76] C. Martin Estefanía, J. del Val Fernández, A. Nieto Sánchez and A. Guerrero Sola, [Palinopsia: first manifestation of a brain metastasis] [Article in Spanish], Neurologia 11 (1996), 357-358.

[77] A.J. Mooney, P. Carey, M. Ryan and P. Bofin, Parasagittal parieto-occipital meningioma with visual hallucinations, Am J Ophthalmol 59 (1965), 197-205.

[78] M.T. Benson and I.G. Rennie, Formed hallucination in the hemianopic field, Postgrad Med J 65 (1989), 756-757.

[79] E. Kasten, E. Muller-Oehring, D. Poggel and B.A. Sabel, [Chronic visual hallucinations and illusions following brain lesions. A single case study], Fortschr Neurol Psychiatr 66 (1998), 49-58.

[80] T.M. Freiman, R. Surges, V.I. Vougioukas, U. Hubbe, J. Talazko, J. Zentner, J. Honegger and A. Schulze-Bonhage, Complex visual hallucinations (Charles Bonnet syndrome) in visual field defects following cerebral surgery. Report of four cases, J Neurosurg 101 (2004), 846-853.

[81] K.M. Faber and L.N. Johnson, Hallucinating the past: a case of spontaneous and involuntary recall of long-term memories: perspectives on the hemispheric organization of visual memory, J Neurol 250 (2003), 55-62.

[82] M. Waragai, Y. Takaya and M. Hayashi, [Complex visual hallucinations in the hemianopic field following an ischemic lesion of the occipitotemporal base - confirmation of the lesion by MRI and speculations on the pathophysiology], No To Shinkei 48 (1996), 371-376.

[83] S. Beniczky, S. Keri, E. Voros, A. Ungurean, G. Benedek, Z. Janka and L. Vecsei, Complex hallucinations following occipital lobe damage, Eur J Neurol 9 (2002), 175-176.

[84] D.G. Cogan, Visual hallucinations as release phenomena, Albrecht Von Graefes Arch Klin Exp Ophthalmol 188 (1973), 139-150.

[85] N. Nagaratnam, S. Virk and O. Brdarevic, Musical hallucinations associated with recurrence of a right occipital meningioma, Br J Clin Pract 50 (1996), 56-57.

[86] M. Cole, When the left brain is not right the right brain may be left: report of personal experience of occipital hemianopia, J Neurol Neurosurg Psychiatry 67 (1999), 169-173.

[87] S.W. Anderson and M. Rizzo, Hallucinations following occipital lobe damage: the pathological activation of visual representations, J Clin Exp Neuropsychol 16 (1994), 651-663.

[88] M.E. Ritsema and M.A. Murphy, Palinopsia From Posterior Visual Pathway Lesions Without Visual Field Defects, $J$ Neuroophthalmol 27 (2007), 115-117.

[89] D. Kompf, H.F. Piper, B. Neundorfer and H. Dietrich, [Palinopsia (visual preservation) and cerebral polyopia - clinical analysis and computed tomographic findings], Fortschr Neurol Psychiatr 51 (1983), 270-281.

[90] R. Huna-Baron and M.J. Kupersmith, Cerebral polyopia, neuroimaging localization, Neuro-Ophthalmology 24 (2000), 267-271.

[91] M. Manford and F. Andermann, Complex visual hallucinations. Clinical and neurobiological insights, Brain $121(\mathrm{Pt}$ 10) (1998), 1819-1840.

[92] A. Ardila, M. Botero and J. Gomez, Palinopsia and visual allesthesia, Int J Neurosci 32 (1987), 775-782.

[93] T.H. Johnson, Visual hallucinations accompanying organic lesions of the brain, with special reference to their value as localizing phenomena, Trans Am Iphthalmol Soc 31 (1933), 244-394.

[94] D.J. Werring and C.D. Marsden, Visual hallucinations and palinopsia due to an occipital lobe tuberculoma, J Neurol Neurosurg Psychiatry 66 (1999), 684. 
[95] V.U. Bhaskaran and R. Prabhu, A case of simultaneous infarctions involving brain and heart, Cal Med J1 (2003), 1-5.

[96] J. Dejerine, P. Sollier and E. Auscher, Deux cas d'hémianopsie homonyme par lésions de l'écorce du lobe occipital, Archives de Physiologie 2 (1890), 177-192.

[97] M.S. Vaphiades, G.G. Celesia and M.G. Brigell, Positive spontaneous visual phenomena limited to the hemianopic field in lesions of central visual pathways, Neurology 47 (1996), 408-417.

[98] M. Critchley, Types of visual perseveration: "paliopsia" and "illusory visual spread", Brain 74 (1951), 267-299.

[99] B.R. Ott and J.L. Saver, Unilateral amnesic stroke. Six new cases and a review of the literature, Stroke 24 (1993), 10331042.

[100] G.N. Dutton, Cognitive vision, its disorders and differential diagnosis in adults and children: knowing where and what things are, Eye 17 (2003), 289-304.
[101] A. Kanzaki, S. Endo, M. Takatani and M. Tanimizu, Neuroimages of migrainous infarction with metamorphopsia and visual hallucination, Neurological Medicine (Japanese) $\mathbf{5 3}$ (2000), 580-582.

[102] L. Manning and R. Campbell, Optic aphasia with spared action naming: a description and possible loci of impairment, Neuropsychologia 30 (1992), 587-592.

[103] D.N. Levine, R.B. Mani and R. Calvanio, Pure agraphia and Gerstmann's syndrome as a visuospatial-language dissociation: an experimental case study, Brain Lang 35 (1988), 172-196.

[104] G. Cocchini, A. Bartolo and P. Nichelli, Left Ipsilesional Neglect for Visual Imagery: A Mental Image Generation Impairment? Neurocase 12 (2006), 197-206(10). 


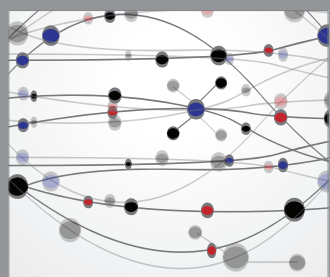

The Scientific World Journal
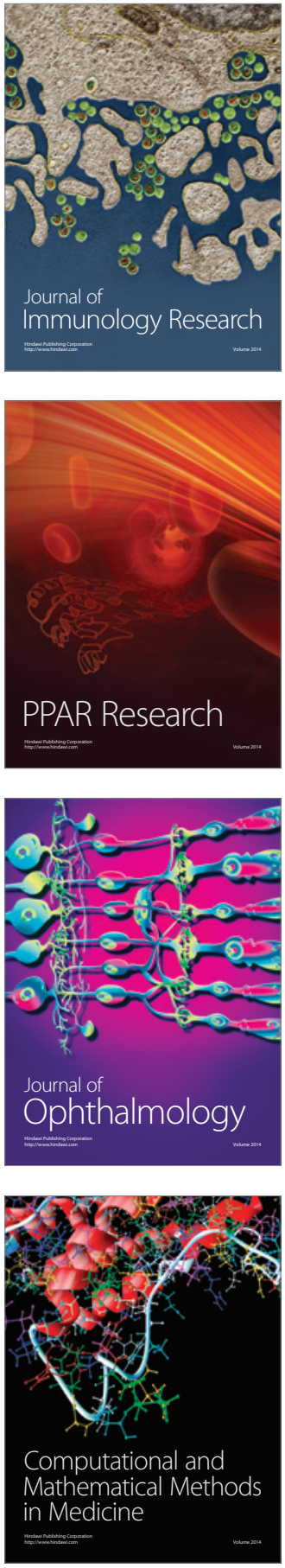

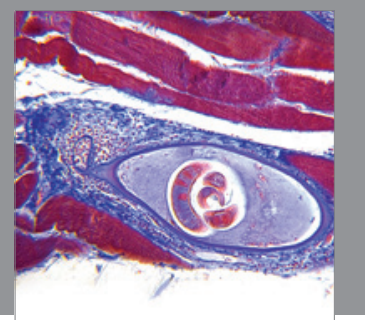

Gastroenterology

Research and Practice
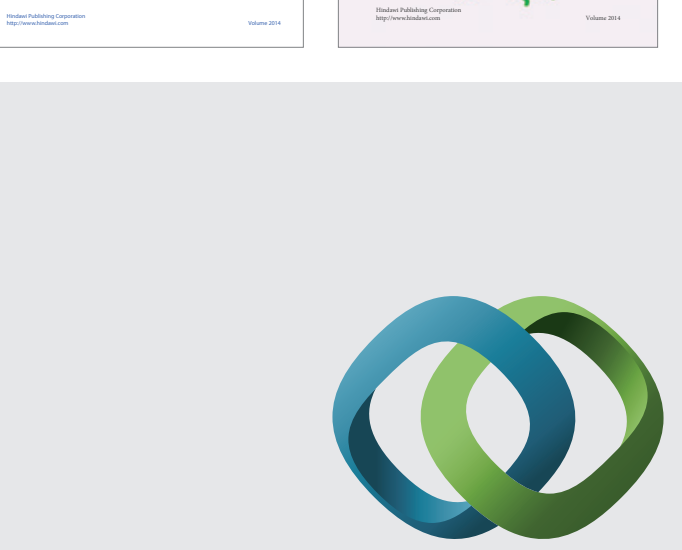

\section{Hindawi}

Submit your manuscripts at

http://www.hindawi.com
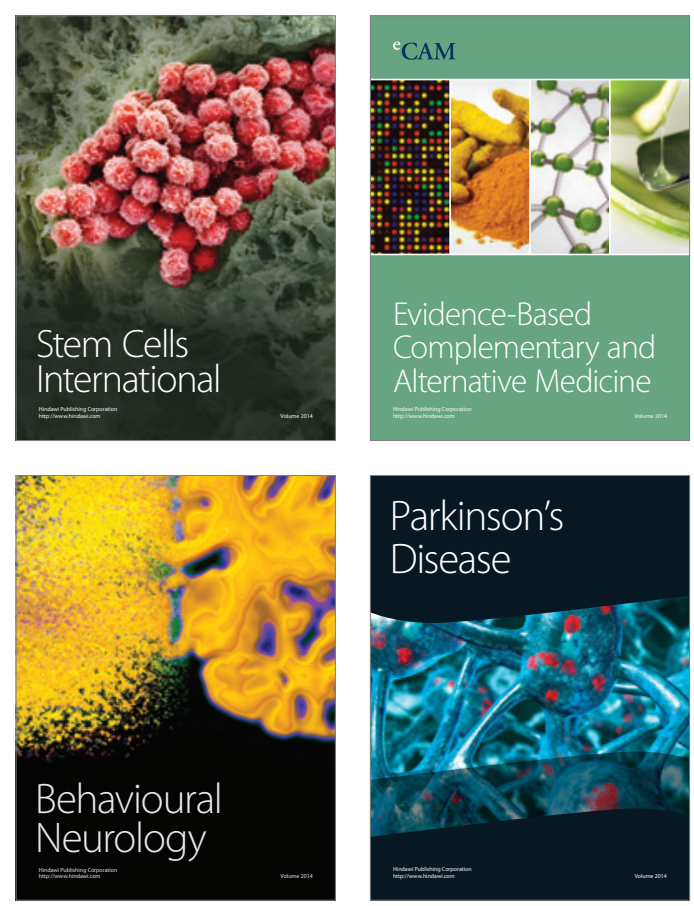

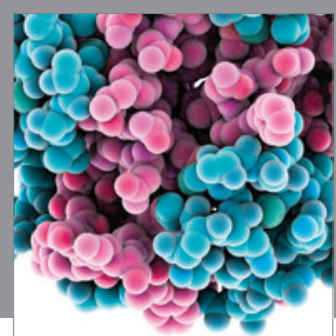

Journal of
Diabetes Research

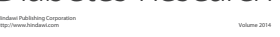

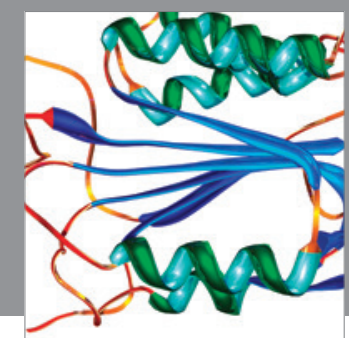

Disease Markers
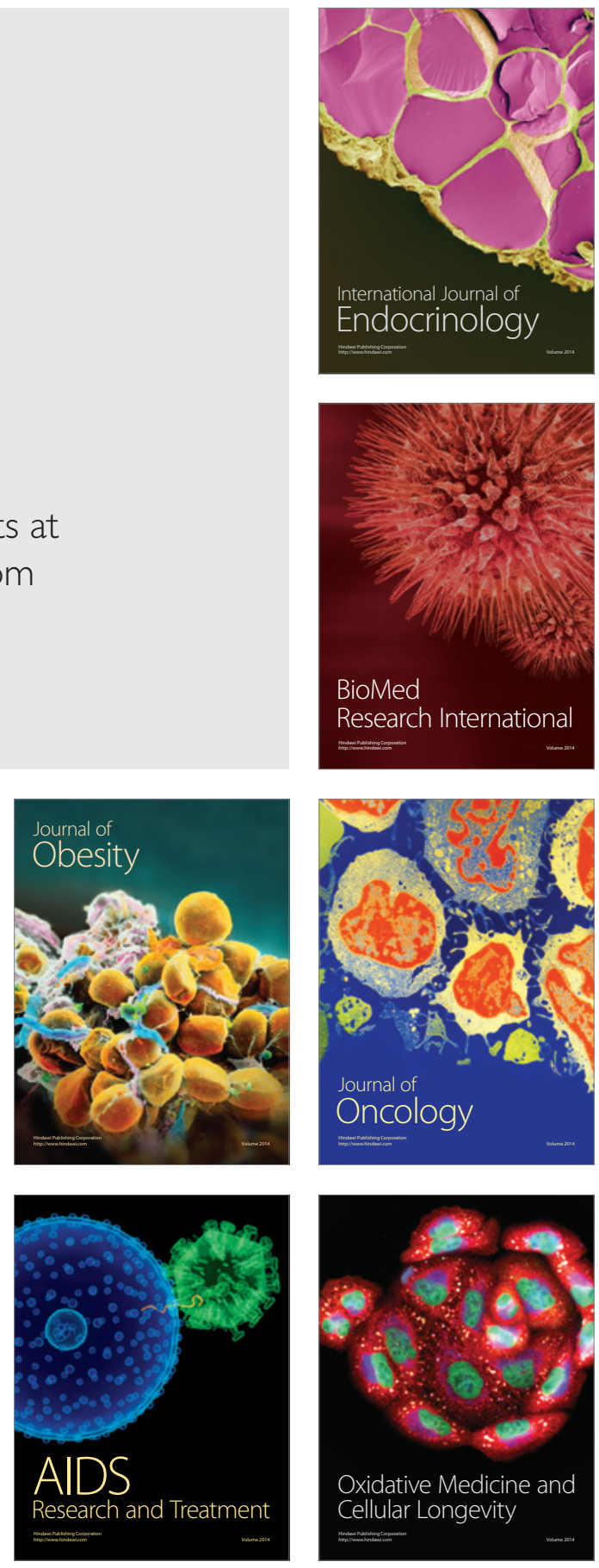